\title{
Transcriptome analysis revealed gene regulatory network involved in PEG-induced drought stress in Tartary buckwheat (Fagopyrum Tararicum)
}

\author{
Juan Huang ${ }^{\text {Equal first author, } 1}{ }^{1}$, Qijiao Chen ${ }^{\text {Equal frrst author, }{ }^{1} \text {, Yuping Rong }}{ }^{1}$, Bin Tang ${ }^{1}$, li wei Zhu ${ }^{1}$, Rongrong Ren ${ }^{1}$, Taoxiong \\ Shi ${ }^{1}$, Qing Fu Chen ${ }^{\text {Corresp. } 1}$ \\ 1 Guizhou Normal University, Research Center of Guizhou Buckwheat Engineering and Technology, Research Center of Buckwheat Industry Technology,
Guiyang, Guizhou, P.R. China \\ Corresponding Author: Qing Fu Chen \\ Email address: cqf1966@163.com
}

Tartary buckwheat is a nutritious pseudo-cereal crop that is resistant to abiotic stresses, such as drought. However, the buckwheat's mechanisms for responding to drought stress remains unknown. We investigated the changes in physiology and gene expression under drought stress, which was simulated by treatment with polyethylene glycol (PEG). Five physiological indexes, namely MDA content, $\mathrm{H}_{2} \mathrm{O}_{2}$ content, CAT activity, SOD activity, and POD activity, were measured over time after 20\% PEG treatment. All indexes showed dramatic changes in response to drought stress. A total of 1,190 differentially expressed genes (DEGs) were identified using RNA-seq and the most predominant were related to a number of stress-response genes and late embryogenesis abundant (LEA) proteins. DEGs were gathered into six clusters and were found to be involved in the ABA biosynthesis and signal pathway based on hierarchical clustering and GO and KEGG pathway enrichment. Transcription factors, such as NAC and bZIP, also took part in the response to drought stress. We determined an ABA-dependent and ABA-independent pathway in the regulation of drought stress in Tartary buckwheat. To the best of our knowledge, this is the first transcriptome analysis of drought stress in Tartary buckwheat, and our results provide a comprehensive gene regulatory network of this crop in response to drought stress. 


\section{Transcriptome analysis revealed gene regulatory}

2 network involved in PEG-induced drought stress in

3 Tartary buckwheat (Fagopyrum Tararicum)

4

5 Juan Huang ${ }^{* 1}$, Qijiao Chen ${ }^{* 1}$, Yuping Rong ${ }^{1}$, Bin Tang ${ }^{1}$, Liwei Zhu¹, Rongrong Ren ${ }^{1}$, Taoxiong

6 Shi ${ }^{1}$, Qingfu Chen ${ }^{1}$

7

$8{ }^{1}$ Research Center of Guizhou Buckwheat Engineering and Technology, Research Center of

9 Buckwheat Industry Technology, Guizhou Normal University, Guiyang, Guizhou, P.R. China 10

11 Corresponding Author:

12 Qingfu Chen ${ }^{1}$

13 Baoshanbeilu NO. 116, Guiyang, 550001, Guizhou, P.R. China

14 Email address: cqf1966@163.com

15 "These authors contributed equally to this work. 


\section{Abstract}

17 Tartary buckwheat is a nutritious pseudo-cereal crop that is resistant to abiotic stresses, such as drought. However, the buckwheat's mechanisms for responding to drought stress remains unknown. We investigated the changes in physiology and gene expression under drought stress, which was simulated by treatment with polyethylene glycol (PEG-6000). Five physiological indexes, namely MDA content, $\mathrm{H}_{2} \mathrm{O}_{2}$ content, CAT activity, SOD activity, and POD activity, were measured over time after 20\% PEG treatment. All indexes showed dramatic changes in response to drought stress. A total of 1,190 differentially expressed genes (DEGs) were identified using RNA-seq and the most predominant were related to a number of stress-response genes and late embryogenesis abundant (LEA) proteins. DEGs were classified into six clusters and were found to be involved in the ABA biosynthesis and signal pathway based on hierarchical clustering and GO and KEGG pathway enrichment. Transcription factors, such as NAC and bZIP, also took part in the response to drought stress. We determined an ABA-dependent and ABA-independent pathway in the regulation of drought stress in Tartary buckwheat. To the best of our knowledge, this is the first transcriptome analysis of drought stress in Tartary buckwheat, and our results provide a comprehensive gene regulatory network of this crop in response to

\section{Introduction}

Tartary buckwheat (Fagopyrum Tararicum), also called bitter buckwheat, is a pseudo-cereal crop belonging to the genus Fagopyrum Mill, Polygonaceae (Ohnishi 1998). Tartary buckwheat has become popular for its rich nutritional composition that includes a high content of flavonoids, resistant starches, crude fibers, proteins, and vitamins, which are all shown to have health benefits (Frias et al., 2011; Qin et al., 2010; Zhu 2016). Tartary buckwheat is also highly adaptable to adverse soil and climatic conditions and shows a very strong tolerance or resistance to the adverse environment and abiotic stresses, including drought, low temperatures, and acid soils (Zhang et al., 2017).

Drought is a major meteorological disaster in agriculture of China and leads to the reduction of crop yield and quality (Xu et al., 2015). A series of physiological responses occur in Tartary buckwheat under water stress (Chen et al., 2008; Xiang et al., 2013), including a decrease of Chlorophyll a, chlorophyll b, and total chlorophyll content and a significant increase in proline accumulation (Xiang et al., 2013). Photosynthesis, transpiration, stomatal conductance, and yield decrease but intercellular $\mathrm{CO}_{2}$ concentration increases (Xiang et al., 2013). Peroxidases (POD) activity, superoxide dismutase (SOD) activity, catalase (CAT) activity, hydrogen peroxide $\left(\mathrm{H}_{2} \mathrm{O}_{2}\right)$ content, and soluble protein and proline content increase significantly and relative water content decreases rapidly in Tartary buckwheat under drought stress (Chen et al., 2008; Xiang et 53 al., 2013). 
55

56

57

58

59

60

61

62

63

64

65

66

67

68

69

70

71

72

73

74

75

76

77

78

79

80

81

82

83

84

85

86

87

88

89

90

91

92

93

94

Plants respond to water-deficit conditions with a series of processes at the physiological, cellular, and molecular levels to improve their stress tolerance (Shinozaki \& Yamaguchi-Shinozaki 2007). At the molecular level, the induction or suppression of candidate genes function to regulate plant damage and the stress response (Shinozaki \& Yamaguchi-Shinozaki 2007). Many droughtinduced genes have been identified using large scale sequencing or gene function studies relating to functional proteins (transporters, detoxification enzymes, late embryogenesis abundant (LEA) proteins, proteases, key enzymes for osmolyte biosynthesis, and detoxification enzymes) and regulatory proteins (transcription factors (TFs), protein kinases, protein phosphatases, and proteins involved in signal biosynthesis and transduction, such as ABA) (Abdel-Ghany et al., 2020; Du et al., 2010; Frey et al., 2012; Li et al., 2008; Roychoudhury et al., 2013; Shi et al., 2020; Shinozaki \& Yamaguchi-Shinozaki 2007). Previous studies have supported that LEA proteins were involved in protecting higher plants from damage caused by environmental stresses, especially drought, by acting as antioxidants and membrane and protein stabilizers during water stress (Hong-Bo et al., 2005; Tunnacliffe \& Wise 2007). Genes encoding $\mathrm{H}_{2} \mathrm{O}_{2}$ scavengers, such as CAT, SOD and POD were also involved in the adaptation of plant drought responses (Luna et al., 2005; Molina-Rueda et al., 2013; Xiao et al., 2020). Many TFs, including $A B R E 2, R D 29 B, R D 20 A, M Y B 2, M B C 2, R D 26, D R E B 1 D, H B, R D 22, D R E B 2$, NAC, and bZIP TFs (Fu et al., 2016; Lee et al., 2010; Roychoudhury et al., 2013; Shinozaki \& YamaguchiShinozaki 2007; Yamaguchi-Shinozaki \& Shinozaki 2005) regulated plant drought resistance through two regulatory pathways: the ABA-dependent pathway and ABA-independent pathway (Abdel-Ghany et al., 2020; Du et al., 2010; Frey et al., 2012; Li et al., 2008; Roychoudhury et al., 2013; Shi et al., 2020; Shinozaki \& Yamaguchi-Shinozaki 2007).

Previous studies on Tartary buckwheat focused on changes in gene regulation under drought and identified some drought-inducible TFs, including three MYB family genes (FtMYB9, FtMYB10, and FtMYB13), eight NAC family genes (from FtNAC2 to FtNAC9), two bZIP family genes (FtbZIP5 and FtbZIP83), and a HLH family gene (FtbHLH3) (Deng et al., 2019; Gao et al., 2016; Gao et al., 2017; Huang et al., 2018; Li et al., 2019; Li et al., 2020; Sun et al., 2019; Yao et al., 2017). The transcriptome analysis of Tartary buckwheat under drought stress has not been reported despite the reports of other transcriptome analyses related to seed development (Huang et al., 2017; Liu et al., 2018). Many drought-inducible genes are unknown and the regulation mechanism of Tartary buckwheat under drought stress is still under investigation.

We examined the dynamic changes of five physiological indexes under drought stress, which was simulated by $20 \%$ polyethylene glycol (PEG-6000) treatment. We then performed a comprehensive transcriptome analysis using high throughput RNA-seq. The identification of differentially expressed genes (DEGs), the hierarchical cluster, GO enrichment, and KEGG enrichment was then analyzed. Genes in ABA biosynthesis and signal transduction were significantly enriched; thereafter $\mathrm{ABA}$ content after drought treatment was measured. The expression patterns of 18 important DEGs were verified using qRT-PCR. We proposed an ABA- 
95

96

97

98

99

100

101

102

103

104

105

106

107

108

109

110

111

112

113

114

115

116

117

118

119

120

121

122

123

124

125

126

127

128

129

130

131

132

133

dependent and ABA-independent pathway in the regulation of drought stress for Tartary

buckwheat. This is the first known transcriptome analysis under drought stress for Tartary buckwheat, and provides a comprehensive gene regulatory network of Tartary buckwheat in response to drought stress.

\section{Materials \& Methods}

\section{Plant materials and growth conditions}

A widely cultivated Tartary buckwheat variety, Jinqiao No. 2, was selected for its high stability and adaptability ( $\mathrm{Li}$ et al., 2011). Seeds were surface sterilized with a $10 \% \mathrm{H}_{2} \mathrm{O}_{2}$ solution, rinsed three times with double distilled water $\left(\mathrm{ddH}_{2} \mathrm{O}\right)$, and placed on wet filter papers for two days to accelerate germination. The uniformly sprouted seeds were moved to rolls of papers soaked with $0 \%, 10 \%, 20 \%$, and $30 \%$ PEG- 6000 solutions, respectively, to evaluate the genotypes after drought stress. After 48 hours, the root length of the seedlings was compared across the four treatments. Three biological replicates were performed, and at least 7 seedlings were included in each biological replicate.

The uniformly sprouted seeds were moved to rolls of papers soaked with double distilled water when seedlings grew two true leaves, which occurred around day seven, in order to measure the physiological indexes and transcriptome sequencing. The seedlings with consistent growth were treated with $20 \%$ PEG based on the literature (Abdel-Ghany et al., 2020). Samples were taken at $0 \mathrm{~h}, 1 \mathrm{~h}, 3 \mathrm{~h}$, and $6 \mathrm{~h}$, frozen in liquid nitrogen, and stored at $-80^{\circ} \mathrm{C}$. Three biological replicates were performed, and 40 seedlings were included in each biological replicate.

\section{Physiological indexes measurement}

A total of 5 physiological indexes, namely malondialdehyde (MDA) content, $\mathrm{H}_{2} \mathrm{O}_{2}$ content, CAT activity, SOD activity, and POD activity were measured, using determination kits (Sino Best Bio-Technology Co. Ltd, China) following the manufacturer's instructions. Three biological replicates were included for each treatment, and 2 technical replicates were included for each biological replicate.

\section{Total RNA isolation and transcriptome sequencing}

Total RNA was isolated using the plant RNA purification kit (TianGen Biotech Co. LTD, China), according to the manufacturer's instructions. The purified RNA samples were treated with Dnase I for 20 minutes to digest the genomic DNA. The quality and quantity of RNA were determined using the NanoDrop 2000 micro spectrophotometer, the Agilent 2100 Bioanalyzer, and the Agilent RNA 6000 Nano Kit. A total of 12 RNA samples were used to construct the library, and high-throughput sequencing was performed using the Illumina 4000 System (Illumina Inc., USA) with a read length of $150 \mathrm{bp}$ and paired-end method. The raw data were accessible via the accession number CRA003335 at Genome Sequence Archive 
134 (https://bigd.big.ac.cn/gsa/) and bioproject PRJCA003569

135 (https://bigd.big.ac.cn/bioproject/browse/PRJCA003569).

136

137

Reads mapping and analysis of differentially expressed genes (DEGs)

138

Raw reads were filtered using Trimmomatic-0.38 (Bolger et al., 2014) with the following

139 settings: trimmomatic-0.38.jar PE -threads 30 input_forward.fq.gz input_reverse.fq.gz

140 output_forward_paired.fq.gz output_forward_unpaired.ff.gz output_reverse_paired.fq.gz

141 output_reverse_unpaired.fq.gz LEADING:3 TRAILING:3 SLIDINGWINDOW:4:20 MINLEN:140. Quality control of all of the acquired clean reads was conducted using FastQC

143 (https://github.com/s-andrews/FastQC) with the following parameters: fastqc -t $30 *$.paired. Then the clean reads were mapped to the Tartary buckwheat genome data using Hisat2 (Kim et al., 2015) with the following parameters: hisat2 -x genome -1 output_forward_paired -2 output_reverse_paired -S *.sam. To evaluate the replicate reproducibility, the genetic FPKM value was calculated using cufflinks (Trapnell et al., 2010). PCA and Pearson correlation analyses were performed in R language (https://www.R-project.org/).

DEGs were identified with the DESeq2 package in R language, with the false discovery rate of (padj) $<0.05$ and absolute value of $\log _{2}$ Ratio $\geq 1$ as the thresholds. Gene annotation was performed by a local BLASP against the NR database, with the threshold of 1e-5. GO items were enriched by agriGO (http://bioinfo.cau.edu.cn/agriGO/) and the KEGG pathway was enriched by KOBAS 3.0 (http://kobas.cbi.pku.edu.cn/kobas3/?t=1). The significantly enriched GO items and KEGG pathways were visualized in $\mathrm{R}$ language.

\section{Measurement of lycopene, zeaxanthin, and ABA content}

High performance liquid chromatography was used to measure the content of lycopene, zeaxanthin, and ABA. For the measurement of lycopene and zeaxanthin, approximately $0.300 \mathrm{~g}$ well-ground samples were precisely weighed and placed in a $5 \mathrm{~mL}$ brown volumetric flask. Then $5 \mathrm{~mL} 0.1 \%$ bht-ethanol solution was added and oscillated for $5 \mathrm{~min}$. The mixture was oscillated at $200 \mathrm{r} / \mathrm{min}$ for $4 \mathrm{~h}$ at room temperature in the dark. A $0.1 \%$ bht-ethanol solution was added to keep the volume of mixture at $10 \mathrm{~mL}$. The mixture was subsequently centrifuged at $4000 \mathrm{r} / \mathrm{min}$ for $10 \mathrm{~min}$ and $1 \mathrm{~mL}$ of the supernatant was filtered using a $0.22 \mu \mathrm{m}$ Millipore filter, and the solution was collected in a $1.5 \mathrm{~mL}$ brown sample bottle. Lycopene and zeaxanthin were tested using Agilent HPLC-1100 with a DAD detector and Thermopylae C18 chromatographic column. The test conditions were as follows: column temperature, $25^{\circ} \mathrm{C}$; injection volume, $20 \mathrm{ul}$; flow rate, $1.0 \mathrm{ml} / \mathrm{min}$; mobile phase, acetonitrile: methanol $=65: 35(\mathrm{~V}: \mathrm{V})$ isoelution.

Samples were well-ground and weighed to approximately $0.300 \mathrm{~g}$ to measure for ABA. Three $\mathrm{mL}$ were precooled and $80 \%$ methanol was added and mixed by oscillation. The mixture was sealed and stored overnight at $4{ }^{\circ} \mathrm{C}$, then centrifuged at $5000 \mathrm{r} / \mathrm{min}$ at $4{ }^{\circ} \mathrm{C}$ for $10 \mathrm{~min}$. The supernatant was taken, and the residue was extracted ultrasonically with precooled $80 \%$ 
174

175

176

177

178

179

180

181

182

183

184

185

186

187

188

189

190

191

192

193

194

195

196

197

198

199

200

201

202

203

204

205

206

207

208

209

210

211

212

methanol at $4{ }^{\circ} \mathrm{C}$ for $30 \mathrm{~min}$. This procedure was repeated twice and the supernatants were combined. The combined supernatant was blown to the aqueous phase with nitrogen at $4{ }^{\circ} \mathrm{C}$. Three $\mathrm{mL}$ petroleum ether was added three times for decolorization, the aqueous phase was extracted with ethyl acetate three times then combined with the ethyl acetate phase and blown dry at $4{ }^{\circ} \mathrm{C}$ with nitrogen. The acetic acid solution $(\mathrm{pH}=3.5)$ was added and purified in a SEPPakC18 column. The eluent was eluted with methanol at room temperature and reduced to dry. ABA was tested by Agilent HPLC-1100 using the VWD detector and Agilent C18 chromatographic column $(250 * 4.6 \mathrm{~mm} ; 5 \mu \mathrm{L})$. The testing conditions were as follows: column temperature, $25{ }^{\circ} \mathrm{C}$; injection volume, $10 \mathrm{ul}$; wavelength: $254 \mathrm{~nm}$; flow rate, $1.0 \mathrm{ml} / \mathrm{min}$; mobile phase, methanol: aqueous acetic acid solution $(\mathrm{pH}=3.6)$ isoelution.

\section{Quantitative RT-PCR (qRT-PCR) analysis}

The transcriptome results were verified by qRT-PCR. A total of 17 DEGs in the ABA biosynthesis and signal transduction pathways were selected. Actin was used as the inner reference gene. Primer3Plus (http://www.primer3plus.com/cgi-bin/dev/primer3plus.cgi) was used to select gene-specific primers (Table S1). qRT-PCR was performed using the SYBR ${ }^{\circledR}$ Premix Ex Taq ${ }^{\mathrm{TM}}$ II kit (Takara Biomedical Technology (Beijing) Co., Ltd., China) on an ABI 7500 Fast Real-Time PCR system (Applied Biosystems, USA) following the manufacturer's instructions, with three technical replicates. qRT-PCR results were calculated using the $2^{-\Delta \Delta \mathrm{Ct}}$ method.

\section{Results}

\section{Investigation of drought tolerance of Tartary buckwheat}

To determine the drought tolerance of Tartary buckwheat, we measured the root length of Tartary buckwheat seedlings after treatment with $0 \%, 10 \%, 20 \%$, and 30\% PEG. This method has been widely used to induce a water deficit in plants (Abdel-Ghany et al., 2020). The results are shown in Figure 1. The seedlings treated with 10\% PEG grew less root hair compared with those treated with $0 \%$ PEG; however, the root length showed no significant difference between the two treatments. The roots were dramatically shortened after treatment with $20 \%$ PEG compared to those treated with $0 \%$ PEG and 10\% PEG. The roots did not grow well after treatment with $30 \%$ PEG, leading to the loss of approximately $50 \%$ of the seedlings. Seedlings treated with 20\% PEG showed significant reductions in growth and root length, therefore, we used a $20 \%$ PEG treatment for the drought stress experiment.

\section{Physiological changes of Tartary buckwheat seedlings under drought stress}

We measured five physiological indexes involved in the drought stress response, namely MDA content, $\mathrm{H}_{2} \mathrm{O}_{2}$ content, CAT activity, SOD activity, and POD activity. There indexes were measured at four time points after being treated with $20 \%$ PEG and five physiological indexes showed significant alterations (Figure 2). The $\mathrm{H}_{2} \mathrm{O}_{2}$ content was significantly increased after $1 \mathrm{~h}$ 
213 treatment, but remained nearly unchanged after $3 \mathrm{~h}$ and $6 \mathrm{~h}$ treatments. The MDA content was

214 significantly increased after $1 \mathrm{~h}$ treatment and was significantly decreased afterwards. CAT

215 activity showed an opposite trend to that of the $\mathrm{H}_{2} \mathrm{O}_{2}$ content, which was significantly decreased

216 after $1 \mathrm{~h}$ treatment, but remained almost unchanged after $3 \mathrm{~h}$ and $6 \mathrm{~h}$ treatments. The SOD and

217 POD activity showed an opposite trend to that of the MDA content, with a significant decrease

218 after $1 \mathrm{~h}$ treatment, and a significant increase afterwards.

219

220

\section{High-throughput RNA-Seq of Tartary buckwheat seedlings under drought stress}

221

We performed high-throughput RNA-Seq after 1, 3, and $6 \mathrm{~h}$ PEG treatments to obtain the

222 transcriptome dynamics of Tartary buckwheat seedlings after drought tolerance. The $0 \mathrm{~h}$

223 treatment was the control. Three biological replicates were included for each treatment. We

224 performed Pearson's rank correlation analysis to evaluate the repeatability and reproducibility of

225 the transcriptome data. The values of Pearson $\mathrm{R}$ between the two samples from the same

226

227

228

229 biological replicates were higher than those of different biological replicates (Fig. S1), indicating that the biological replicates had good repeatability.

230

We obtained 44,303,640 to $62,033,328$ raw reads and 42,564,428 to 59,376,546 clean reads for each library (Table 1). The quality of sequencing was high with the Q30 ranging from $92.53 \%$ to

231

232

233

234

235

236

237

238

239

240

241

242

243

244

245

246

247

248

249

250

251

252 93.29\%. The genome data of Tartary buckwheat (Zhang et al., 2017) was used as the reference data for mapping, and we successfully mapped $74.49 \%$ to $75.45 \%$ of the clean reads to the predicted coding sequences (Table 1). A total of 27,490 genes were identified, with 24,433, 24,462, 24,508, 24,271, 24,680, 24,531, 24,617, 24,531, 24,398, 24,694, 24,553, and 24,263 genes identified in the libraries of PEG0h-1, PEG0h-2, PEG0h-3, PEG1h-1, PEG1h-2, PEG1h-3, PEG3h-1, PEG3h-2, PEG3h-3, PEG6h-1, PEG6h-2, and PEG6h-3, respectively (Table 1). We mapped 25,895, 25,982, 25,957, and 26,033 genes in the treatment of PEG0h, PEG1h, PEG3h, and PEG6h, respectively (Figure 3A). We also performed a principal component analysis (PCA) and the results showed that samples were similar from the same time point after PEG treatment; whereas samples from the time point after the PEG treatment were not (Figure 3B).

\section{Analysis of DEGs of Tartary buckwheat seedlings under drought stress}

We analyzed DEGs using the pair-wise comparison of samples after different treatment times with $20 \%$ PEG as the control to illustrate the transcriptome changes of Tartary buckwheat seedlings under drought stress. One thousand one hundred-ninety genes were up or down regulated by drought stress, among which 177, 558, and 315 DEGs were up-regulated in $1 \mathrm{~h}$ vs. $0 \mathrm{~h}, 3 \mathrm{~h}$ vs. $0 \mathrm{~h}$, and $6 \mathrm{~h}$ vs. $0 \mathrm{~h}$, whereas 36, 176, and 382 DEGs were down-regulated in $1 \mathrm{~h}$ vs. 0 h, 3 h vs. 0 h, and 6 h vs. 0 h (Figure 4 and Table S2). Of these DEGs, 86 genes were co-upregulated at $1 \mathrm{~h}$ vs. $0 \mathrm{~h}, 3 \mathrm{~h}$ vs. $0 \mathrm{~h}$, and $6 \mathrm{~h}$ vs. $0 \mathrm{~h}$, whereas 6 genes were co-down-regulated at 1 h vs. $0 \mathrm{~h}, 3 \mathrm{~h}$ vs. $0 \mathrm{~h}$, and $6 \mathrm{~h}$ vs. $0 \mathrm{~h}$. There were 53, 115, and 6 genes up-regulated in the comparisons of $1 \mathrm{~h}$ vs. $0 \mathrm{~h}, 3 \mathrm{~h}$ vs. $0 \mathrm{~h}, 3 \mathrm{~h}$ vs. $0 \mathrm{~h}$, and $6 \mathrm{~h}$ vs. $0 \mathrm{~h}$, and $1 \mathrm{~h}$ vs. $0 \mathrm{~h}$ and $6 \mathrm{~h}$ vs. $0 \mathrm{~h}$, respectively, whereas 14,77 , and 0 genes were down-regulated in the comparisons of $1 \mathrm{~h}$ vs. $0 \mathrm{~h}$ 
253

254

255

256

257

258

259

260

261

262

263

264

265

266

267

268

269

270

271

272

273

274

275

276

277

278

279

280

281

282

283

284

285

286

287

288

289

290

291

292

and $3 \mathrm{~h}$ vs. $0 \mathrm{~h}, 3 \mathrm{~h}$ vs. $0 \mathrm{~h}$ and $6 \mathrm{~h}$ vs. $0 \mathrm{~h}$, and $1 \mathrm{~h}$ vs. $0 \mathrm{~h}$ and $6 \mathrm{~h}$ vs. $0 \mathrm{~h}$, respectively. The remaining genes were differentially regulated at one time point or showed no obvious expression patterns after PEG treatment (Figure 4). It worth to mention that circadian affect gene

regulation. Previous study has suggested that more than one third expected DEGs were classified as clock-controlled genes comparing Arabidopsis sampled at time 0, 0.5, and 1 hours (Hsu \& Harmer 2012). Thus, some fraction of the DEGs identified in our study might not be drought inducible genes, but circadian regulated genes.

The top 20 URGs and DRGs were analyzed after being exposed to drought stress for $1 \mathrm{~h}, 3 \mathrm{~h}$, or $6 \mathrm{~h}$ (Table 2, Table 3, and Table 4), among which many stress responsive genes were identified, including FtPinG0005419000.01 (annotated to dehydrin Rab18) and FtPinG0009412200.01 (annotated to carotenoid oxygenase). Interestingly, 5 LEA proteins (FtPinG0002083100.01, FtPinG0005679700.01, FtPinG0004425400.01, FtPinG0001202200.01, and FtPinG0000702400.01) were up-regulated at the third time point of the PEG treatments, suggesting the LEA proteins played crucial roles in response to drought stress. We found that that genes related to the oxidation-reduction process and reactive oxygen species biosynthesis were suppressed at the $1 \mathrm{~h}$ PEG treatment. These genes included two genes encoding peroxidase (FtPinG0007824100.01 and FtPinG0003282600.01) and one gene encoding allene oxide synthase (FtPinG0002376300.01), suggesting the biosynthesis of the protective enzyme, POD, was suppressed at a molecular level.

\section{Functional category of DEGs of Tartary buckwheat under drought stress}

We performed a hierarchical cluster analysis based on the gene expression pattern, with gene function enrichment based on GO annotation, to determine the biological function of the drought-responsive-genes. Six clusters (C1-C6) were identified (Figure 5 and Table S2). C1 included 352 genes that were mildly down-regulated at $1 \mathrm{~h}$ and $3 \mathrm{~h}$ PEG, and were seriously down-regulated at $6 \mathrm{~h}$ PEG. A total of $11 \mathrm{GO}$ biological processes were significantly enriched in this cluster, most of which were related to $\mathrm{H}_{2} \mathrm{O}_{2}$ metabolism and catabolism, reactive oxygen species metabolism, and oxidative stress responses. $\mathrm{C} 2$ included 189 genes that were upregulated after PEG treatment; however, there was no significantly enriched GO biological process in this cluster. $\mathrm{C} 3$ included 87 genes that were down-regulated at $1 \mathrm{~h}$ and $3 \mathrm{~h} \mathrm{PEG}$, but up-regulated at $6 \mathrm{~h}$ PEG. A total of $14 \mathrm{GO}$ biological processes were significantly enriched in this cluster, most of which were related to cell wall biogenesis, including xyloglucan metabolism, cell wall polysaccharide metabolism, and hemicellulose metabolism. C4 included 291 genes that were up-regulated at $1 \mathrm{~h}$ and $3 \mathrm{~h}$ PEG, but down-regulated at $6 \mathrm{~h} \mathrm{PEG}$, which showed an opposite expression pattern compared to $\mathrm{C} 3$. A total of four GO biological processes were significantly enriched in this cluster, and were related to lipid localization and phospholipid transport. C5 included 214 genes that were up-regulated at $1 \mathrm{~h}$ and $3 \mathrm{~h}$ PEG, but down-regulated at $6 \mathrm{~h}$ PEG, which showed a similar expression pattern to $\mathrm{C} 4$. A total of $25 \mathrm{GO}$ biological processes were significantly enriched in this cluster, most of which were related to the regulation 
293

294

295

296

297

298

299

300

301

302

303

304

305

306

307

308

309

310

311

312

313

314

315

316

317

318

319

320

321

322

323

324

325

326

327

328

329

330

331

332

of candidate processes, such as regulation of gene expression and regulation of biosynthetic process. C6 included 57 genes that were down-regulated at $1 \mathrm{~h}$ PEG, but slightly up-regulated at $3 \mathrm{~h}$ and $6 \mathrm{~h}$ PEG treatment; however, there was no significantly enriched GO biological processes in this cluster.

We also analyzed the metabolic pathways based on KEGG annotation (Figure 6). Two hundred of the 213 DEGS were annotated to 30 KEGG pathways at $1 \mathrm{~h}$ PEG treatment, in which two pathways, "Carotenoid biosynthesis" and "Plant hormone signal transduction", were significantly enriched (Qvalue $<0.05$, Figure 6A). At 3 h PEG treatment, 702 of 734 DEGS were annotated to $81 \mathrm{KEGG}$ pathways, in which six pathways were significantly enriched, namely "Glutathione metabolism", "Protein processing in endoplasmic reticulum", "alpha-Linolenic acid metabolism", "Carotenoid biosynthesis", "Biosynthesis of secondary metabolites", and "Phenylpropanoid biosynthesis" (Qvalue $<0.05$, Figure 6B). At $6 \mathrm{~h}$ PEG treatment, 634 of the 697 DEGS were annotated to 86 KEGG pathways, in which nine pathways were significantly enriched. The pathways were: "Biosynthesis of secondary metabolites", "Circadian rhythm plant", "alpha-Linolenic acid metabolism", "Phenylpropanoid biosynthesis", "Metabolic pathways", "Flavonoid biosynthesis", "Protein processing in endoplasmic reticulum", "Photosynthesis - antenna proteins", and "Carotenoid biosynthesis" (Qvalue $<0.05$, Figure 6C).

\section{The involvement of ABA in response to drought stress in Tartary buckwheat}

The carotenoid biosynthesis pathway was significantly enriched at all time points of PEG treatment, based on the KEGG results. Three DEGs were included in this pathway, namely two PSY genes (FtPinG0005737800.01 and FtPinG0004637900.01) and one BCH gene (FtPinG0006960700.01) (Figure 7). PSY encodes phytoene synthase that catalyzes geranylgeranyl diphosphate to form phytoene, which is the rate-limiting enzyme in the carotenoid biosynthetic pathway. $\mathrm{BCH}$ encodes $\beta$-carotene hydroxylase of the $\mathrm{P}-450$ monooxygenase family that converts $\beta$-carotene to zeaxanthin by a two-step reaction (Ruiz-Sola \& Rodriguez-Concepcion 2012). We determined the contents of phytoene and zeaxanthin to examine whether the carotenoids took a part in a drought response (Fig. S2). The result showed that neither phytoene nor zeaxanthin content were significantly altered after drought stress, indicating that the carotenoids may not be involved in drought response.

In higher plants, $\mathrm{ABA}$ is synthesized from the cleavage of carotenoid precursors ( $9^{\prime}$-cisviolaxanthin and 9'-cis-neoxanthin), which is likely the key regulatory step in the ABA biosynthetic pathway. The cleavage reaction is catalyzed by 9-cis-epoxycarotenoid dioxygenase (NCED), and produces xanthoxin, which can be converted into ABA via ABA-aldehyde (Chernys \& Zeevaart 2000). It is possible that ABA was involved in drought response rather than carotenoids. Interestingly, the plant hormone signal transduction KEGG pathway was one of the most enriched pathways in our results. Fourteen genes related to ABA biosynthesis and signal transduction were differentially expressed in our dataset (Figure 7), including 4 DEGs

Peer] reviewing PDF | (2020:09:53215:2:0:NEW 15 Feb 2021) 
333 homologous to NCED (FtPinG0009412200.01, FtPinG0006853200.01, FtPinG0003131500.01,

334

335

336

337

338

339

340

341

342

343

344

345

346

347

348

349

350

351

352

353

354

355

356

357

358

359

360

361

362

363

364

365

366

367

368

369

370

371

and FtPinG0000246400.01), 2 DEGs homologous to AREB (FtPinG0002143600.01 and

FtPinG0003196200.01), 5 DEGs homologous to PP2C (FtPinG0007629100.01,

FtPinG0009574600.01, FtPinG0004850700.01, FtPinG0002889200.01, and

FtPinG0006346100.01), 2 DEGs homologous to PYR (FtPinG0001214600.01 and

FtPinG0007802700.01), and 1 DEG homologous to OST1 (FtPinG0003981600.01).

Interestingly, 15 of the 17 DEGs showed highly consistent expression patterns and were classified to C5, with the exception of FtPSY2 (C4) and FtPYR1 (C1) (Table S2). Among these, two AREB homologs, FtPinG0002143600.01 and FtPinG0003196200.01, have been identified and named as FtbZIP83 and FtbZIP5 respectively in Tartary buckwheat previously (Li et al., 2019; Li et al., 2020).

We measured the ABA content of the Tartary buckwheat seedlings after drought stress and the result of the physiological changes was in accordance with the molecular changes; the ABA content was significantly increased after PEG treatment (Figure 7).

\section{Transcription Factors (TFs) in response to drought stress in Tartary buckwheat}

Two hundred fourteen DEGs in C5 were related to the regulation processes, such as regulation of gene expression and biosynthetic process, based on the hierarchical cluster and the enriched GO biological processes, which is indicative of TFs' role in the drought stress response. We analyzed the differentially expressed TFs in the dataset. We found that a total of 174 TFs belonging to 36 TF families were identified as DEGs, among which the most abundant TF families were WRKY (27), NAC (14), MYB (13), C3H (12), bZIP (10), AP2-EREBP (8), DBP (7), HSF (7), Orphans (7), bHLH (6), C2H2 (6), and MYB-related (6) (Figure 8). We then analyzed the expression patterns of the top 6 families and the genes in each TF family had a different expression pattern. Overall, most of the TFs were clustered to C5 (59 TFs), followed by C1 (38 TFs), C4 (32 TFs), and C2 (30 TFs) (Table S2), indicating their potential role on the regulation of definite biological processes. For example, among the 27 WRKY TFs, 10 TFs were clustered to C5, suggesting that they may function in the regulation of biosynthetic process; 7 TFs were clustered to $\mathrm{C} 4$, suggesting they may function in lipid localization and phospholipid transport; $3 \mathrm{TFs}$ were clustered to $\mathrm{C} 1$, suggesting that they may function in $\mathrm{H}_{2} \mathrm{O}_{2}$ metabolism and catabolism, reactive oxygen species metabolism, and oxidative stress response; and $3 \mathrm{TFs}$ were clustered to $\mathrm{C} 3$, suggesting they may function in cell wall biogenesis (Figure 5 and Figure 8). In addition, some TFs, such as FtPinG0002173200.01, FtbZIP83, FtbZIP5, FtPinG0007618600.01, and FtPinG0008274300.01, were homologous to the reported genes within the regulatory network of the drought stress response (Fujita et al., 2004; Lee et al., 2010; Li et al., 2008; Li et al., 2019; Li et al., 2020; Trivedi et al., 2016).

Confirmation of the transcriptome data by qRT-PCR

Peer) reviewing PDF | (2020:09:53215:2:0:NEW 15 Feb 2021) 
372 The expression patterns of 31 DEGs were verified by qRT-PCR, including five LEA protein 373 encoding genes, seven genes in ABA biosynthesis (FtNCED1, FtNCED2, FtNCED3, FtNCED4, 374 FtPSY1, FtPSY2, and FtBCH), 16 genes in ABA signal pathway (FtABRE1, FtABRE2, FtPP2C1, 375 FtPP2C2, FtPP2C3, FtPP2C4, FtPP2C5, FtPYR1, FtPYR2, FtOST1, RD29B, RD26, DREB1D, $376 H B 1, H B 2$, and $R D 22$ ), and three TFs in the ABA-independent pathway (DREB2, RD19, and $377 E R D 1)$. The results showed that the expression patterns obtained by qRT-PCR were highly 378 correlated with those obtained by transcriptome data, with a Pearson R correlation ranging from 3790.76 to 0.98 (Figure 9), suggesting that our transcriptome results were reliable.

380

381

\section{Discussion}

382

383

384

385

386

387

388

389

390

391

392

393

394

395

396

397

398

399

400

401

402

403

404

405

406

407

408

409

410

411

Physiological changes were highly consistent with molecular changes of Tartary buckwheat seedlings under drought stress

ROS accumulate in high amounts when a plant survives oxidative damage or various stresses, such as drought, leading to the damage of the membrane protein and lipids. $\mathrm{H}_{2} \mathrm{O}_{2}$ and MDA content increase rapidly under these conditions and the ROS scavenger enzymatic system, including the activities of SOD, APX, and CAT, are induced as an universal response (Mohammadkhani \& Heidari 2007). We measured five widely used physiological indexes in drought stress response, namely MDA content, $\mathrm{H}_{2} \mathrm{O}_{2}$ content, CAT activity, SOD activity, and POD activity, after $20 \%$ PEG treatment. The results showed that the physiological changes were highly consistent with the molecular changes based on the hierarchical cluster and GO enrichment (biological process) analyses (Figure 2, Figure 5, and Table S2). The $\mathrm{H}_{2} \mathrm{O}_{2}$ and MDA content were significantly increased in the $1 \mathrm{~h}$ PEG treatment, whereas the activity of the protective mechanisms was decreased. Genes related to $\mathrm{H}_{2} \mathrm{O}_{2}$ metabolism and catabolism, and ROS metabolism, were clustered to $\mathrm{C} 1$ and were down-regulated at this time point, suggesting that ROS accumulation and membrane damage immediately occurred after drought stress in Tartary buckwheat. In the $3 \mathrm{~h}$ and $6 \mathrm{~h}$ PEG treatment, the activity of the protective enzymes significantly increased, but the MDA content decreased, indicating that the ROS scavenger system was induced against drought stress. The related genes were clustered to $\mathrm{C} 3$ and $\mathrm{C} 4$, which were associated with cell wall biogenesis and metabolism and lipid localization and phospholipid transport. This suggests that the repair of the ROS and damage to the membrane occurred after the damage to the ROS and membrane.

\section{LEA proteins were involved in drought stress response in Tartary buckwheat}

LEA proteins are a type of low molecular weight protein induced by various abiotic stresses, such as drought, high temperature, and cold (Hong-Bo et al., 2005). A number of studies have reported that genes encoding LEA proteins are induced to maintain the stability of the membranes and proteins and to provide detoxification and alleviation of cellular damage under conditions of dehydration (Roychoudhury et al., 2013; Shi et al., 2020; Shinozaki \& YamaguchiShinozaki 2007; Tunnacliffe \& Wise 2007). In a recent transcriptome comparison of droughtresistant and drought-sensitive Sorghum genotypes, six of the 25 top-induced genes in drought- 
412 resistant genotypes encoded LEA proteins (Abdel-Ghany et al., 2020). Our results were similar 413 (Table 2, Table 3 and Table 4). Twenty-nine up-regulated genes were identified, out of which 414 five genes were annotated to LEA proteins. Among these, four genes were up-regulated at all 415 time points after PEG treatment, and included two genes homologues to LEA protein D-29, one 416 gene homologous to LEA protein 2, and one gene homologous to LEA protein 46. Their 417 expression levels were high, with an absolute fold change of 20 to 90 times, suggesting that LEA proteins were involved in the drought stress response in Tartary buckwheat as well.

422

423

424

425

426

427

428

429

430

431

432

433

434

435

436

437

438

439

440

441

442

443

444

445

446

447

448

449

450

451

\section{Transcriptional regulatory network in ABA-dependent and ABA-independent pathways under drought stress of Tartary buckwheat}

Plants have two regulatory pathways, the ABA-dependent pathway and the ABA-independent pathway, to manage drought responsive genes (Roychoudhury et al., 2013; Shinozaki \& Yamaguchi-Shinozaki 2007). To better understand the ABA-dependent and ABA-independent pathways of Tartary buckwheat under drought stress, a transcriptional regulatory network was constructed based on the previously reported regulatory network and data obtained from our study (Figure 10).

The ABA-dependent pathway under drought stress begins with ABA biosynthesis. ABA biosynthesis begins from the cleavage of carotenoid precursors catalyzed by NCED, which is the key regulatory step in the ABA biosynthetic pathway (Chernys \& Zeevaart 2000; Seo \& Koshiba 2002). We found that four genes (FtNCED1, FtNCED2, FtNCED3, and FtNCED4) encoding NCED and three genes (FtPSY1, FtPSY2, and FtBCH) encoding key enzymes (PSY and BCH) in carotenoid biosynthesis were also up-regulated (Figure 7). All of these genes were found to be induced by drought stress or other abiotic stresses in previous studies (Du et al., 2010; Frey et al., 2012; Li et al., 2008). There are at least five signal pathways that regulate drought stress in the ABA-dependent pathway in plants. The primary pathway is the ABRE2 mediated signal pathway (Roychoudhury et al., 2013; Shinozaki \& Yamaguchi-Shinozaki 2007). We identified two genes homologous to ABRE2 (FtbZIP83 and FtbZIP5), and eight genes (FtPYR1, FtPYR2, FtPP2C1, FtPP2C2, FtPP2C3, FtPP2C4, FtPP2C5, and FtOST1) in the upstream of ABRE2 as DEGs (Figure 7). Among these, FtbZIP83 and FtbZIP5 were reported to improve drought/salt tolerance via an ABA-mediated pathway in Transgenic Arabidopsis recently (Li et al., 2019; Li et al., 2020). ABRE2 binds to the DRE/CRT element in the promoter of RD29B and RD20A to activate their expression in response to stress (Yamaguchi-Shinozaki \& Shinozaki 2005). We identified a gene (FtPinG0004870600.01) homologous to RD29B that was induced under drought stress in Tartary buckwheat, however, the homolog of $R D 20 A$ was not among the identified DEGs (Figure 10). The $A B R E 2$ mediated signal pathway in addition to the $M Y B 2$ and $M B C 2$, $R D 26, D R E B 1 D$, and $H B$ mediated signal pathways are also important constituents in plant ABA-dependent pathway (Roychoudhury et al., 2013; Shinozaki \& Yamaguchi-Shinozaki 2007). We found four homologs of RD26 (FtPinG0002173200.01), DREB1D (FtPinG0008274300.01), and $H B$ (FtPinG0001748800.01 and FtPinG0008157500.01), all of which were up-regulated 
452 under drought stress (Table S2). Though we found no homologs of $M Y B 2$ or $M B C 2$, one gene 453 (FtPinG0002802300.01) homologous to its downstream gene, RD22, was identified in our data. 454 It was down-regulated after drought stress and exhibited a different expression pattern compared 455 to other genes in the ABA-dependent pathway (Table S2 and Figure 5). Sixteen of the 20 DEGs mentioned above showed similar expression patterns and were up-regulated at $1 \mathrm{~h}$ and $3 \mathrm{~h} \mathrm{PEG}$, but down-regulated at $6 \mathrm{~h} \mathrm{PEG}$, and clustered to C5 (Table S2, Figure 5 and Figure 7), with the exception of FtPSY2, FtPYR1, FtPinG0008274300.01 and FtPinG0002802300.01. These results confirmed that genes in ABA-dependent regulatory pathway are co-regulators in response to drought stress in Tartary buckwheat. In addition, changes of ABA content corresponded with the molecular changes, and confirmed the role of $\mathrm{ABA}$ in response to drought stress and tolerance in buckwheat (Figure 7).

There are at least two signal pathways similar to ABA-dependent regulatory pathway, namely the DREB2 mediated signal pathway and the NAC and bZIP TFs mediated signal pathway, to regulate drought stress in the plant ABA-independent pathway (Fu et al., 2016; Roychoudhury et al., 2013; Shinozaki \& Yamaguchi-Shinozaki 2007). DREB2 belongs to the AP2-EREBP TF family and may transcriptionally activate the expression of $R D 19$ and $R D 29 A$ (Lee et al., 2010). We identified the homologues of DREB2 (FtPinG0007618600.01) and $R D 19$ (FtPinG0002253700.01) but were unable to identify the homolog of RD29A (Table S2 and Figure 10). Massive NAC and bZIP family TFs have been reported to function in the ABAindependent pathway by activating $E R D 1$, which encodes a chloroplast-targeted Clp protease regulatory subunit that is induced by water stress in Arabidopsis thaliana (Nakashima et al., 2010). We identified a homolog of ERD1 (FtPinG0001990100.01) which was clustered to C2 based on its expression pattern (Table $\mathbf{S 2}$ and Figure 5). Interestingly, some NAC and bZIP TFs were also clustered to $\mathrm{C} 2$, suggesting they may be co-expressed and in the upstream of $E R D 1$ (Table S2, Figure 5, Figure 8 and Figure 10).

The ABA-dependent and ABA-independent pathways were found to participate in the regulation of drought stress of Tartary buckwheat.

\section{Conclusion}

We investigated the physiological changes and the gene expression changes in a time-course manner under drought stress simulated by $20 \%$ PEG treatment. A total of 1,190 DEGs were identified and the genes encoding LEA proteins were listed on the top up-regulated DEGs. They were grouped into six clusters, in which genes showed definite expression patterns and were involved in specific biological processes based on GO annotation. Further analyses of the ABA and TFs revealed they were also involved in the drought stress response in Tartary buckwheat. We proposed ABA-dependent and ABA-independent pathways in the regulation of drought stress of Tartary buckwheat. This is the first study using a large-scale sequencing method to unravel the transcriptomic changes under drought stress in Tartary buckwheat, which identified 
492

493

494

495

496

497

498

499

500

501

502

503

504

505

506

507

508

509

510

511

512

513

514

515

516

517

518

519

520

521

522

523

524

525

526

527

528

529

530

531

532

533

534

535

536

537

538

539

massive genes and a gene regulatory network in response to drought stress. Our study provides candidate genes for further functional studies.

\section{References}

Abdel-Ghany SE, Ullah F, Ben-Hur A, and Reddy ASN. 2020. Transcriptome Analysis of Drought-Resistant and Drought-Sensitive Sorghum (Sorghum bicolor) Genotypes in Response to PEG-Induced Drought Stress. Int J Mol Sci 21. DOI: 10.3390/ijms21030772.

Bolger AM, Lohse M, and Usadel B. 2014. Trimmomatic: a flexible trimmer for Illumina sequence data. Bioinformatics 30:2114-2120. DOI: 10.1093/bioinformatics/btu170.

Chen P, Zhang DJ, Yu-Hong LI, and Chen T. 2008. Effect of Drought Stress during Seedling Stage on Physiological and Biochemical Traits of Tartary Buckwheat. Acta Agriculturae Boreali-Occidentalis Sinica (China) 17:204-207.

Chernys JT, and Zeevaart JA. 2000. Characterization of the 9-cis-epoxycarotenoid dioxygenase gene family and the regulation of abscisic acid biosynthesis in avocado. Plant Physiol 124:343-353. DOI: 10.1104/pp.124.1.343.

Deng R, Zhao H, Xiao Y, Huang Y, Yao P, Lei Y, Li C, Chen H, and Wu Q. 2019. Cloning, Characterization, and Expression Analysis of Eight Stress-Related NAC Genes in Tartary Buckwheat. Crop Science 59:266-279. DOI: 10.2135/cropsci2018.06.0368.

Du H, Wang N, Cui F, Li X, Xiao J, and Xiong L. 2010. Characterization of the beta-carotene hydroxylase gene DSM2 conferring drought and oxidative stress resistance by increasing xanthophylls and abscisic acid synthesis in rice. Plant Physiol 154:13041318. DOI: $10.1104 / p p .110 .163741$.

Frey A, Effroy D, Lefebvre V, Seo M, Perreau F, Berger A, Sechet J, To A, North HM, and Marion-Poll A. 2012. Epoxycarotenoid cleavage by NCED5 fine-tunes ABA accumulation and affects seed dormancy and drought tolerance with other NCED family members. Plant J 70:501-512. DOI: 10.1111/j.1365-313X.2011.04887.x.

Frias J, Penas E, Szawaranowak D, Zielinski H, and Vidalvalverde. 2011. Evaluation of vitamins B1 and B2, soluble proteins and phytic acid in buckwheat enhanced wheat bread. Polish Journal of Food and Nutrition Sciences 61:29-30.

Fu L, Ding Z, Han B, Hu W, Li Y, and Zhang J. 2016. Physiological Investigation and Transcriptome Analysis of Polyethylene Glycol (PEG)-Induced Dehydration Stress in Cassava. Int J Mol Sci 17. DOI: 10.3390/ijms17030283.

Fujita M, Fujita Y, Maruyama K, Seki M, Hiratsu K, Ohme-Takagi M, Tran LS, YamaguchiShinozaki K, and Shinozaki K. 2004. A dehydration-induced NAC protein, RD26, is involved in a novel ABA-dependent stress-signaling pathway. Plant J 39:863-876. DOI: 10.1111/j.1365-313X.2004.02171.x.

Gao F, Yao H, Zhao H, Zhou J, Luo X, Huang Y, Li C, Chen H, and Wu Q. 2016. Tartary buckwheat FtMYB10 encodes an R2R3-MYB transcription factor that acts as a novel negative regulator of salt and drought response in transgenic Arabidopsis. Plant Physiology \& Biochemistry 109:387-396. DOI: 10.1016/j.plaphy.2016.10.022.

Gao F, Zhou J, Deng RY, Zhao HX, Li CL, Chen H, Suzuki T, Park SU, and Wu Q. 2017. Overexpression of a tartary buckwheat R2R3-MYB transcription factor gene, FtMYB9, enhances tolerance to drought and salt stresses in transgenic Arabidopsis. Journal of Plant Physiology 214:81-90. DOI: 10.1016/j.jplph.2017.04.007. 
540

541

542

543

544

545

546

547

548

549

550

551

552

553

554

555

556

557

558

559

560

561

562

563

564

565

566

567

568

569

570

571

572

573

574

575

576

577

578

579

580

581

582

583

584

585

586

587

588

589

590

Hong-Bo S, Zong-Suo L, and Ming-An S. 2005. LEA proteins in higher plants: structure, function, gene expression and regulation. Colloids Surf B Biointerfaces 45:131-135. DOI: 10.1016/j.colsurfb.2005.07.017.

Hsu PY, and Harmer SL. 2012. Circadian Phase Has Profound Effects on Differential Expression Analysis. PloS one 7:e49853. DOI: 10.1371/journal.pone.0049853.

Huang J, Deng J, Shi T, Chen Q, Liang C, Meng Z, Zhu L, Wang Y, Zhao F, Yu S, and Chen Q. 2017. Global transcriptome analysis and identification of genes involved in nutrients accumulation during seed development of rice tartary buckwheat (Fagopyrum Tararicum). Scientific Reports 7:11792. DOI: 10.1038/s41598-017-11929-Z.

Huang Y, Zhao H, Gao F, Yao P, Deng R, Li C, Chen H, and Wu Q. 2018. A R2R3-MYB transcription factor gene, FtMYB13, from Tartary buckwheat improves salt/drought tolerance in Arabidopsis. Plant Physiology \& Biochemistry 132:238-248. DOI: 10.1016/j.plaphy.2018.09.012.

Kim D, Langmead B, and Salzberg SL. 2015. HISAT: a fast spliced aligner with low memory requirements. Nat Methods 12:357-360. DOI: 10.1038/nmeth.3317.

Lee SJ, Kang JY, Park HJ, Kim MD, Bae MS, Choi HI, and Kim SY. 2010. DREB2C interacts with ABF2, a bZIP protein regulating abscisic acid-responsive gene expression, and its overexpression affects abscisic acid sensitivity. Plant Physiol 153:716-727. DOI: 10.1104/pp.110.154617.

Li F, Vallabhaneni R, and Wurtzel ET. 2008. PSY3, a new member of the phytoene synthase gene family conserved in the Poaceae and regulator of abiotic stress-induced root carotenogenesis. Plant Physiol 146:1333-1345. DOI: 10.1104/pp.107.111120.

Li Q, Wu Q, Wang A, Lv B, Dong Q, Yao Y, Wu Q, Zhao H, Li C, and Chen H. 2019. Tartary buckwheat transcription factor FtbZIP83 improves the drought/salt tolerance of Arabidopsis via an ABA-mediated pathway. Plant Physiology \& Biochemistry 144:312323. DOI: 10.1016/j.plaphy.2019.10.003.

Li Q, Zhao H, Wang X, Kang J, Lv B, Dong Q, Li C, Chen H, and Wu Q. 2020. Tartary Buckwheat Transcription Factor FtbZIP5, Regulated by FtSnRK2.6, Can Improve Salt/Drought Resistance in Transgenic Arabidopsis. Int J Mol Sci 21. DOI: 10.3390/jms21031123.

Li X, Shi X, Gao W, Zhu H, and Cui L. 2011. Analysis of high and stable yield of new Tartary buckwheat variety 'Jinqiaomai2' and utilization prospect. Journal of Agriculture (China) 1:6-10.

Liu M, Ma Z, Zheng T, Sun W, Zhang Y, Jin W, Zhan J, Cai Y, Tang Y, Wu Q, Tang Z, Bu T, Li $C$, and Chen H. 2018. Insights into the correlation between Physiological changes in and seed development of tartary buckwheat (Fagopyrum tataricum Gaertn.). BMC genomics 19:648-648. DOI: 10.1186/s12864-018-5036-8.

Luna CM, Pastori Gm Fau - Driscoll S, Driscoll S Fau - Groten K, Groten K Fau - Bernard S, Bernard S Fau - Foyer $\mathrm{CH}$, and Foyer CH. 2005. Drought controls on $\mathrm{H} 2 \mathrm{O} 2$ accumulation, catalase (CAT) activity and CAT gene expression in wheat. J Exp Bot 56:417-423. DOI: 10.1093/jxb/eri039.

Mohammadkhani N, and Heidari R. 2007. Effects of drought stress on protective enzyme activities and lipid peroxidation in two maize cultivars. Pak J Biol Sci 10:3835-3840. DOI: 10.3923/pjbs.2007.3835.3840.

Molina-Rueda JJ, Tsai CJ, and Kirby EG. 2013. The Populus superoxide dismutase gene family and its responses to drought stress in transgenic poplar overexpressing a pine cytosolic glutamine synthetase (GS1a). PloS one 8:e56421-e56421. DOI: 10.1371/journal.pone.0056421.

Nakashima K, Kiyosue T, Yamaguchi-Shinozaki K, and Shinozaki K. 2010. A nuclear gene, erd1, encoding a chloroplast-targeted Clp protease regulatory subunit homolog is not only induced by water stress but also developmentally up-regulated during senescence

Peer) reviewing PDF | (2020:09:53215:2:0:NEW 15 Feb 2021) 
591

592

593

594

595

596

597

598

599

600

601

602

603

604

605

606

607

608

609

610

611

612

613

614

615

616

617

618

619

620

621

622

623

624

625

626

627

628

629

630

631

632

633

634

635

636

637

638

639

640

641

in Arabidopsis thaliana. Plant Journal 12:851-861. DOI: 10.1046/j.1365-

313X.1997.12040851.x.

Ohnishi O. 1998. Search for the wild ancestor of buckwheat III. The wild ancestor of cultivated common buckwheat, and of tatary buckwheat. Economic Botany 52:123-133. DOI: 10.1007/bf02861199.

Qin P, Wang Q, Shan F, Hou Z, and Ren G. 2010. Nutritional composition and flavonoids content of flour from different buckwheat cultivars. International Journal of Food Science and Technology 45:951-958. DOI: 10.1111/j.1365-2621.2010.02231.x.

Roychoudhury A, Paul S, and Basu S. 2013. Cross-talk between abscisic acid-dependent and abscisic acid-independent pathways during abiotic stress. Plant Cell Rep 32:985-1006. DOI: 10.1007/s00299-013-1414-5.

Ruiz-Sola MA, and Rodriguez-Concepcion M. 2012. Carotenoid biosynthesis in Arabidopsis: a colorful pathway. Arabidopsis Book 10:e0158. DOI: 10.1199/tab.0158.

Seo M, and Koshiba T. 2002. Complex regulation of ABA biosynthesis in plants. Trends Plant Sci 7:41-48. DOI: 10.1016/s1360-1385(01)02187-2.

Shi H, He X, Zhao Y, Lu S, and Guo Z. 2020. Constitutive expression of a group 3 LEA protein from Medicago falcata (MfLEA3) increases cold and drought tolerance in transgenic tobacco. Plant Cell Rep 39:851-860. DOI: 10.1007/s00299-020-02534-y.

Shinozaki K, and Yamaguchi-Shinozaki K. 2007. Gene networks involved in drought stress response and tolerance. J Exp Bot 58:221-227. DOI: 10.1093/jxb/erl164.

Sun W, Ma Z, Chen H, and Liu M. 2019. MYB Gene Family in Potato (Solanum tuberosum L.): Genome-Wide Identification of Hormone-Responsive Reveals Their Potential Functions in Growth and Development. Int J Mol Sci 20. DOI: 10.3390/ijms20194847.

Trapnell C, Williams BA, Pertea G, Mortazavi A, Kwan G, Van Baren MJ, Salzberg SL, Wold BJ, and Pachter L. 2010. Transcript assembly and quantification by RNA-Seq reveals unannotated transcripts and isoform switching during cell differentiation. Nature Biotechnology 28:511-515. DOI: 10.1038/nbt.1621.

Trivedi DK, Gill SS, and Tuteja N. 2016. Abscisic Acid (ABA): Biosynthesis, Regulation, and Role in Abiotic Stress Tolerance: Wiley - VCH Verlag GmbH \& Co. KGaA.

Tunnacliffe A, and Wise MJ. 2007. The continuing conundrum of the LEA proteins. Naturwissenschaften 94:791-812. DOI: 10.1007/s00114-007-0254-y.

Xiang DB, Peng LX, Zhao JL, Zou L, and Song C. 2013. Effect of drought stress on yield, chlorophyll contents and photosynthesis in tartary buckwheat (Fagopyrum tataricum). Journal of Food Agriculture and Environment 11:1358-1363.

Xiao H, Wang C, Khan N, Chen M, Fu W, Guan L, and Leng X. 2020. Genome-wide identification of the class III POD gene family and their expression profiling in grapevine (Vitis vinifera L). BMC genomics 21. DOI: 10.1186/s12864-020-06828-Z.

Xu K, Yang D, Yang H, Li Z, Qin Y, and Shen Y. 2015. Spatio-temporal variation of drought in China during 1961-2012: A climatic perspective. Journal of Hydrology 526:253-264. DOI: $10.1016 / j . j h y d r o l .2014 .09 .047$.

Yamaguchi-Shinozaki K, and Shinozaki K. 2005. Organization of cis-acting regulatory elements in osmotic- and cold-stress-responsive promoters. Trends Plant Sci 10:88-94. DOI: 10.1016/j.tplants.2004.12.012.

Yao PF, Li CL, Zhao XR, Li MF, Zhao HX, Guo JY, Cai Y, Chen H, and Wu Q. 2017. Overexpression of a Tartary Buckwheat Gene, FtbHLH3, Enhances Drought/Oxidative Stress Tolerance in Transgenic Arabidopsis. Frontiers in Plant Science. DOI: 10.3389/fpls.2017.00625.

Zhang L, Li X, Ma B, Gao Q, Du H, Han Y, Li Y, Cao Y, Qi M, Zhu Y, Lu H, Ma M, Liu L, Zhou J, Nan C, Qin Y, Wang J, Cui L, Liu H, Liang C, and Qiao Z. 2017. The Tartary Buckwheat Genome Provides Insights into Rutin Biosynthesis and Abiotic Stress Tolerance. Mol Plant 10:1224-1237. DOI: 10.1016/j.molp.2017.08.013.

Peer] reviewing PDF | (2020:09:53215:2:0:NEW 15 Feb 2021) 
642 Zhu F. 2016. Buckwheat starch: Structures, properties, and applications. Trends in Food

643 Science and Technology 49:121-135. DOI: 10.1016/j.tifs.2015.12.002.

644 


\section{Figure 1}

Figure 1. Investigation of drought tolerance of Tartary buckwheat.

A - D, the morphology of Tartary buckwheat seedlings treated by the PEG of $0 \%, 10 \%, 20 \%$, $30 \%$. E, root length quantification of Tartary buckwheat seedlings treated by the PEG of $0 \%$, $10 \%, 20 \%, 30 \%$. 

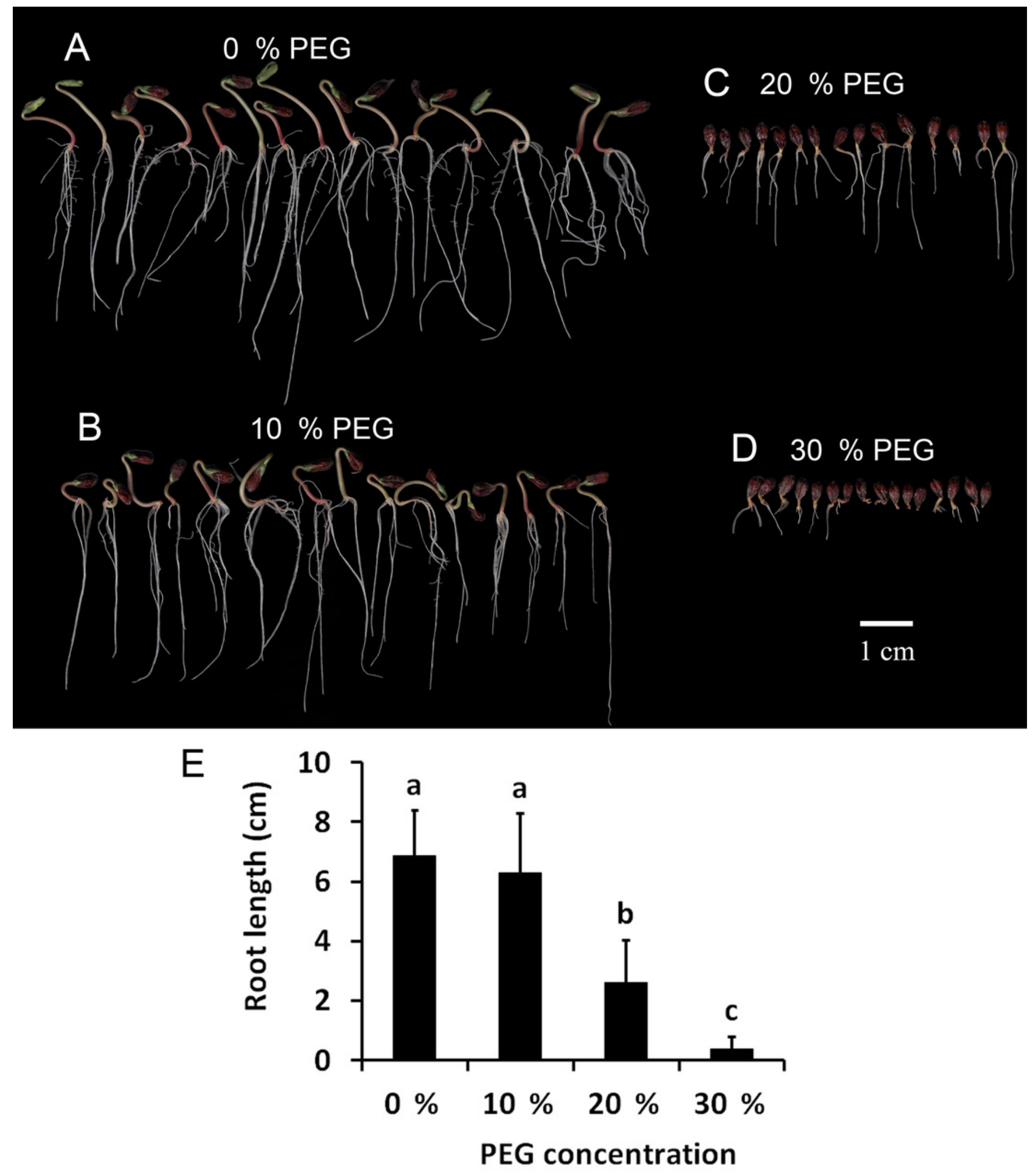
Figure 2

Figure 2. Physiological changes of Tartary buckwheat seedlings under drought stress.

A, $\mathrm{H}_{2} \mathrm{O}_{2}$ content after PEG treatment. B, MDA content after PEG treatment. C, CAT activity after PEG treatment. D, SOD activity after PEG treatment. E, POD activity after PEG treatment. 

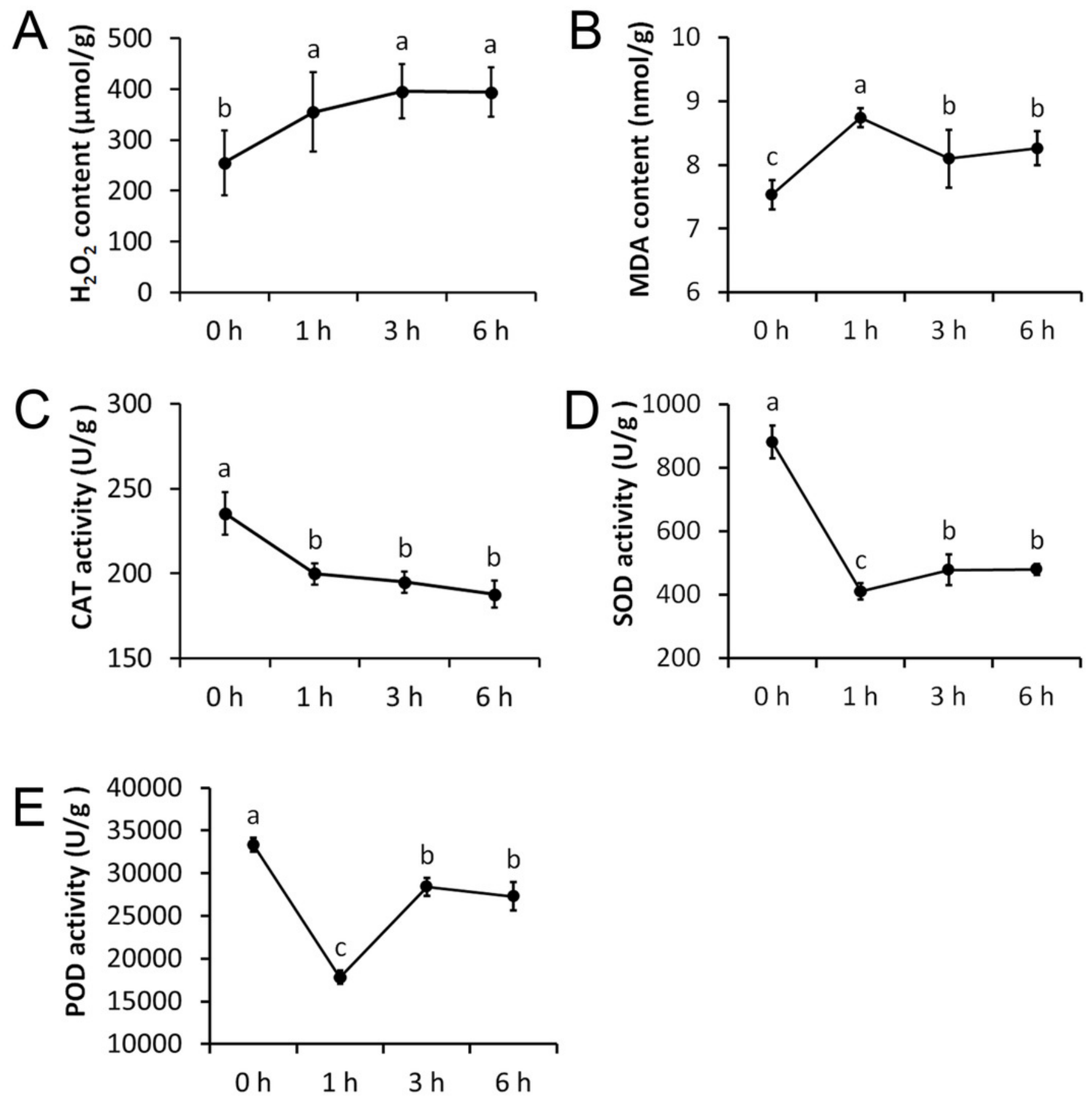
Figure 3

Figure 3. Global analysis of gene expression after $0 \mathrm{~h}, 1 \mathrm{~h}, 3 \mathrm{~h}$, and $6 \mathrm{~h}$ treatment of PEG.

$A$, the number of mapped genes in control and 3 treatments. B, Principle component analysis (PCA) of all samples after PEG treatment.
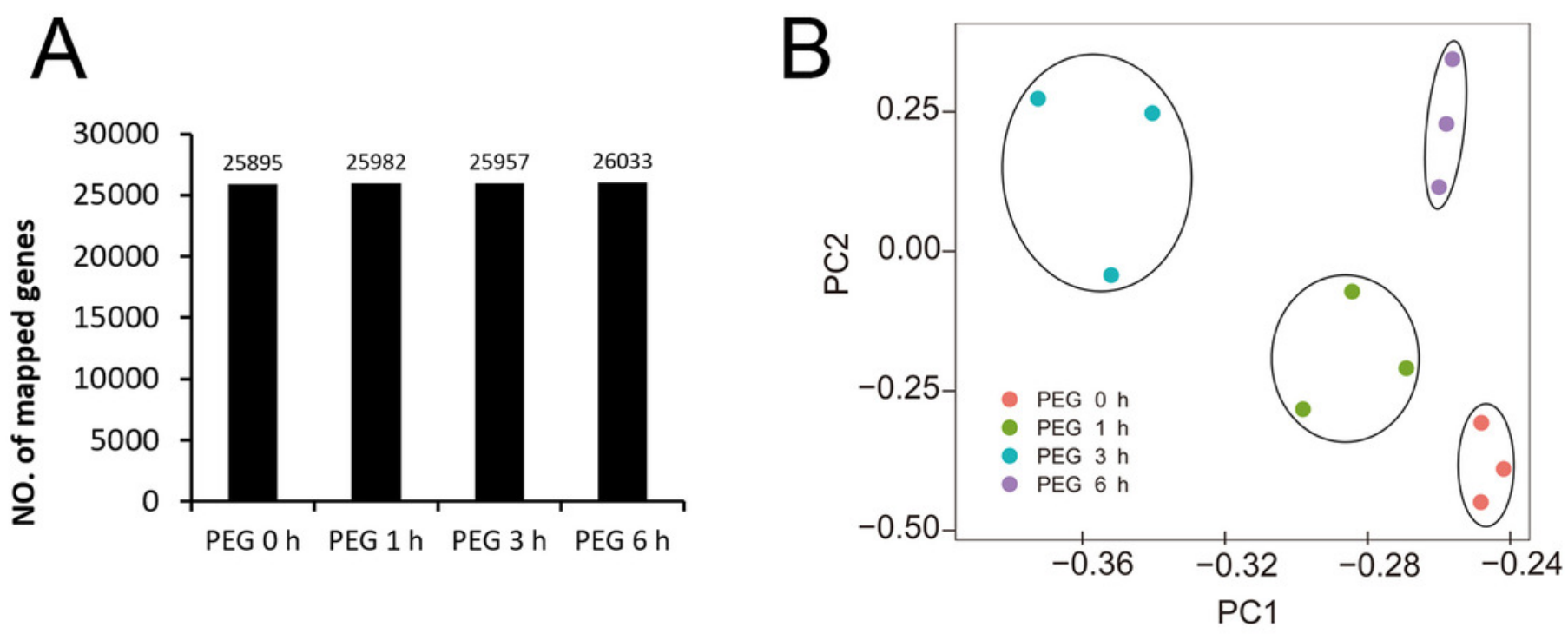


\section{Figure 4}

Figure 4. Analysis of DEGs of Tartary buckwheat seedlings under drought stress.

Pink or dark blue bars represented the number of genes that were up or down regulated in comparison of $1 \mathrm{~h}, 3 \mathrm{~h}$, and $6 \mathrm{~h}$ treatment $20 \%$ PEG with the control. Black bars represented the number of co-regulated genes under PEG treatment. Red or green dots represented genes that were up or down regulated in all three time point of PEG treatment. Orange or light blue dots represented genes that were up or down regulated in at least two time point of PEG treatment. Black dots represented genes that were differentially regulated in one time point or showed no obvious expression patterns after PEG treatment. URG, up-regulated gene; DRG, down-regulated gene.

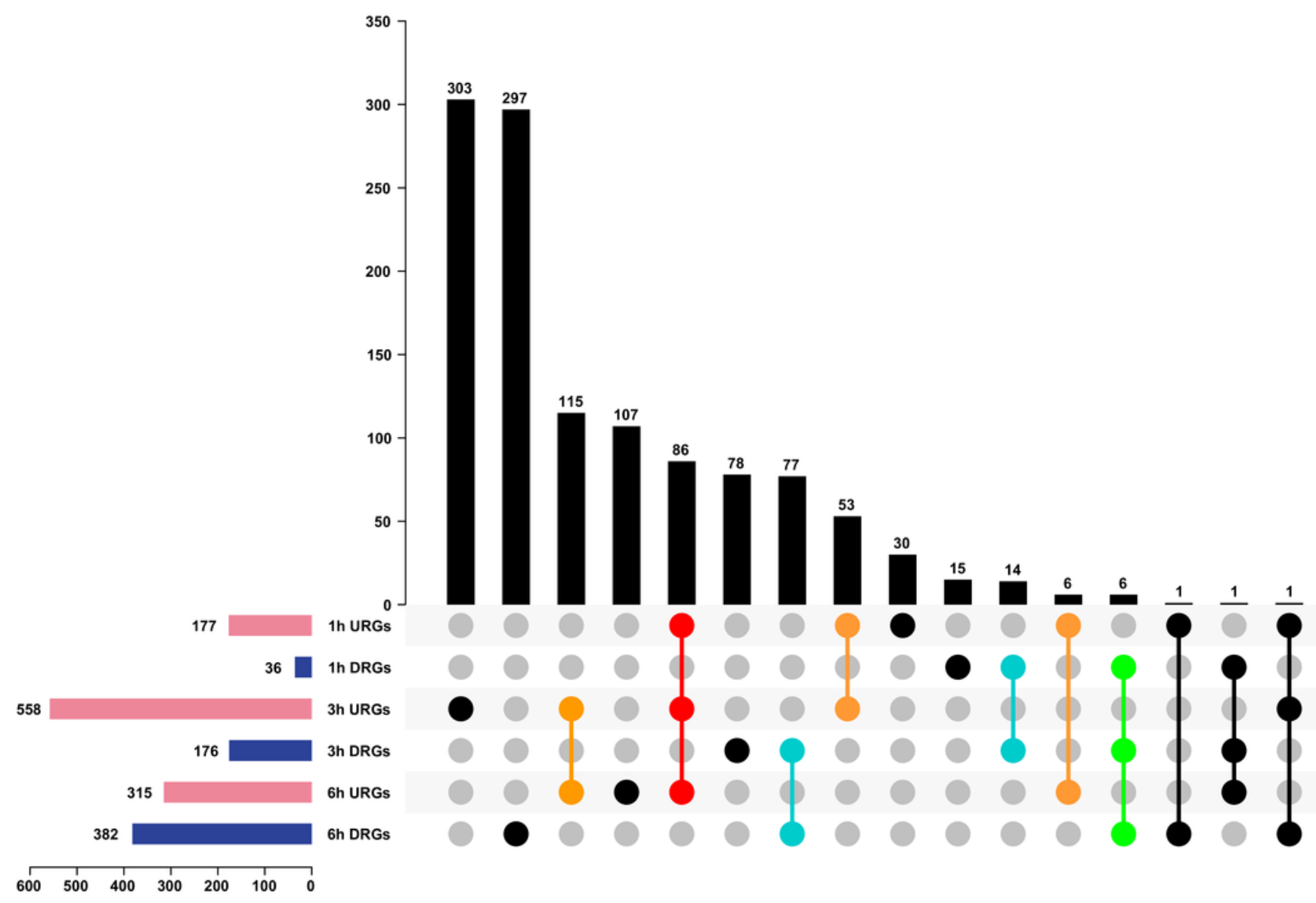


Figure 5

Figure 5. Functional category of DEGs of Tartary buckwheat under drought stress.

A, Hierarchical cluster of the drought-responsive-DEGs. B-G, Expression patterns of the 6

clusters correspondent to the Hierarchical cluster result. Six main clusters were presented as

$\mathrm{C} 1$ - C6. H, GO biological processes significantly enriched in 6 clusters, based on GO

annotation. Missing GO-slim was represented by grey color.

A
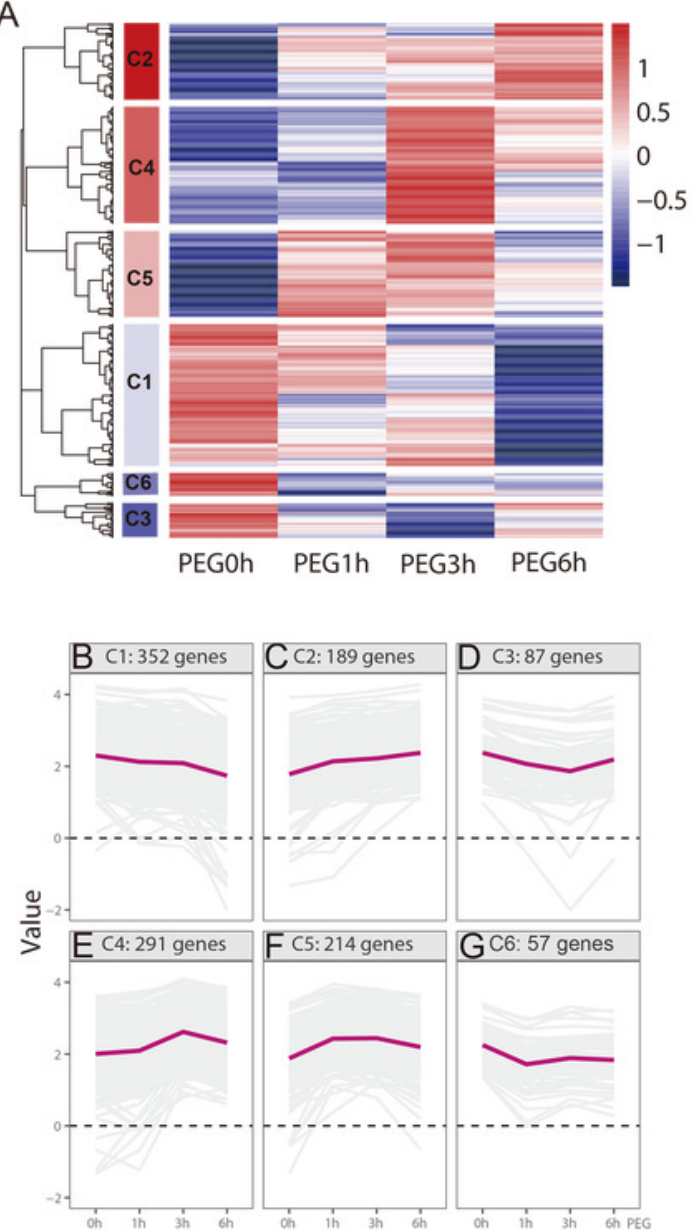

$\mathrm{H}$

GO:0042743

GO:0042744

GO:0072593

GO:0055114

GO:0044248

GO:0006950

GO:0009056

GO:0044710

GO:0050896

GO:0044699

GO:0010411

GO:0010383

GO:0010410

GO:0044036

GO:0071554

GO:0006073

GO:0044042

GO:0044262

GO:0044264

GO:0005976

GO:0044085

GO:0005975

GO:0071840

GO:0010876

GO:0006869

GO:0015914

GO:0015748

GO:0032774

GO:0031323

GO:0050789

GO:0080090

GO:2000112

GO:0097659

GO:0065007

GO:0031326

GO:0010468

GO:0009889

GO:0050794

GO:1903506

GO:2001141

GO:0051252

GO:0006355

GO:0010556

GO:0006351

GO:005117

G.00625

GO.0019222

G. 01813

(1) 0019438

GO:0019438

$\mathrm{C} 1 \mathrm{C} 2 \mathrm{C} 3 \mathrm{C} 4 \mathrm{C} 5 \mathrm{C} 6$

hydrogen peroxide metabolic process

hydrogen peroxide catabolic process

reactive oxygen species metabolic process

response to oxidative stress

cellular catabolic process

response to stress

response to stress

single-organism metabolic process

ringe-organism metabc

single-organism process

xyloglucan metabolic process

cell wall polysaccharide metabolic process

hemicellulose metabolic process

cell wall macromolecule metabolic process

cell wall biogenesis

cell wall organization or biogenesis

cellular glucan metabolic process

glucan metabolic process

cellular carbohydrate metabolic process

cellular polysaccharide metabolic process

polysaccharide metabolic process

cellular component biogenesis

carbohydrate metabolic process

cellular component organization or biogenesis

lipid localization

lipid transport

phospholipid transport

organophosphate ester transport

RNA biosynthetic process

regulation of cellular metabolic process

regulation of biological process

metabolic process

regulation of cellular macromolecule biosynthetic process

nucleic acid-templated transcription

biological regulation

regulation of cellular biosynthetic process

regulation of gene expression

regulation of nucleobase-containing compound metabolic process regulation of biosynthetic process

regulation of cellular process

regulation of nucleic acid-templated transcription

regulation of RNA biosynthetic process

regulation of transcription, DNA-templated

regulation of macromolecule biosynthetic process

transcription, DNA-templated
regulation of nitrogen compound metabolic process

regulation of macromolecule metabolic process

regulation of metabolic process

nucleobase-containing compound biosynthetic process

nucleobase-containing compound biosyn
aromatic compound biosynthetic process

aromatic compound biosynthetic process
organic cyclic compound biosynthetic process 
Figure 6

Figure 6. Top 20 enrichment KEGG pathways under drought stress.

A, Top 20 enrichment KEGG pathways at $1 \mathrm{~h}$ under drought stress. B, Top 20 enrichment KEGG pathways at $3 \mathrm{~h}$ under drought stress. C, Top 20 enrichment KEGG pathways at $6 \mathrm{~h}$ under drought stress. 

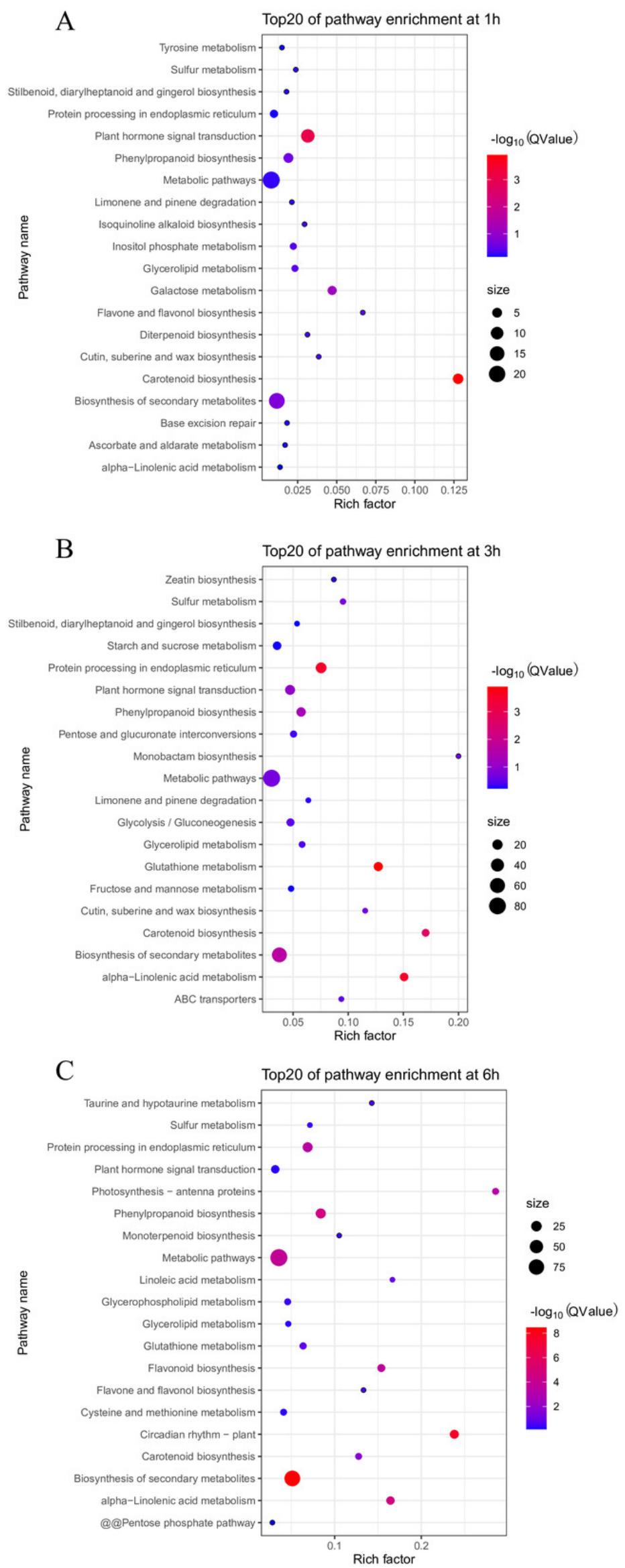

Peer) reviewing PDF | (2020:09:53215:2:0:NEW 15 Feb 2021) 


\section{Figure 7}

Figure 7. ABA biosynthesis and signal transduction pathway were involved in response to drought stress.

A, Overview of ABA biosynthesis and signal transduction pathway. The red rounded boxes represented substrates or products. The orange ellipse represented the enzymes or proteins. $B, C, D, F, G, H$, and I, Heatmap represented the expression patterns of genes correspondent to the enzymes or proteins. The values were normalized to $\log _{2}(F P K M)$. E, ABA content after PEG treatment. 
A

Carotenoid biosynthesis

ABA biosynthesis

ABA signal transduction
GGPP

PSY

$\downarrow$

phytoene

$\mathrm{BCH}$

v

zeaxanthin

I

NCED

I
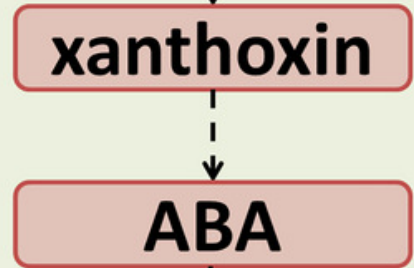

$\downarrow$

PYR

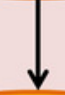

PP2C

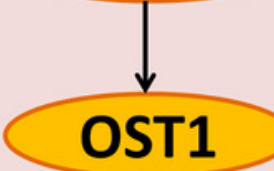

OST1

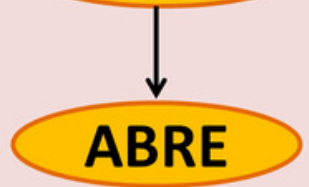

$\begin{array}{lllll} & 0.5 & 0 & -0.5 & -1\end{array}$
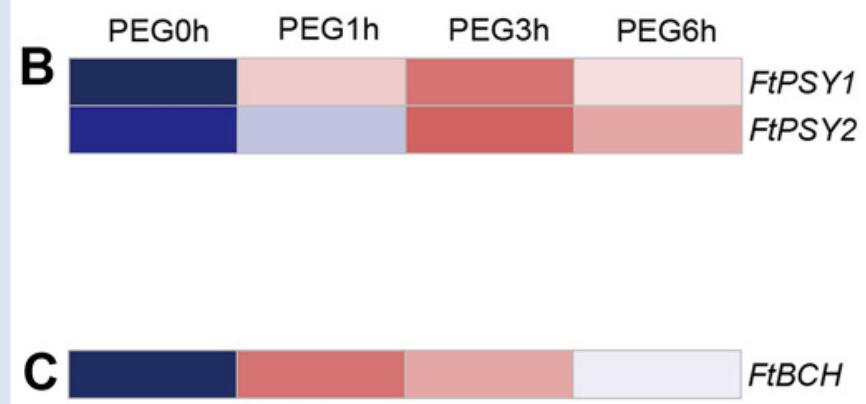

D
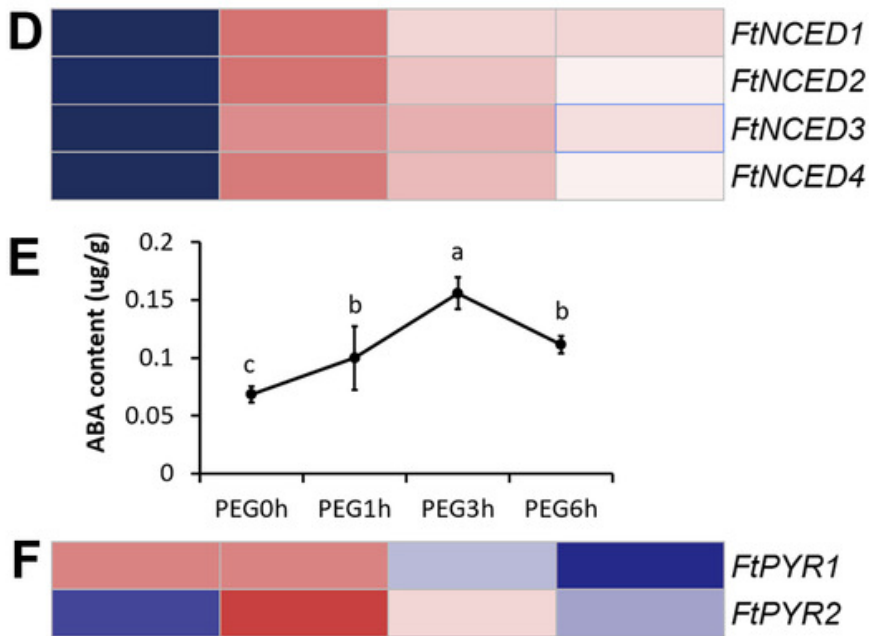

G

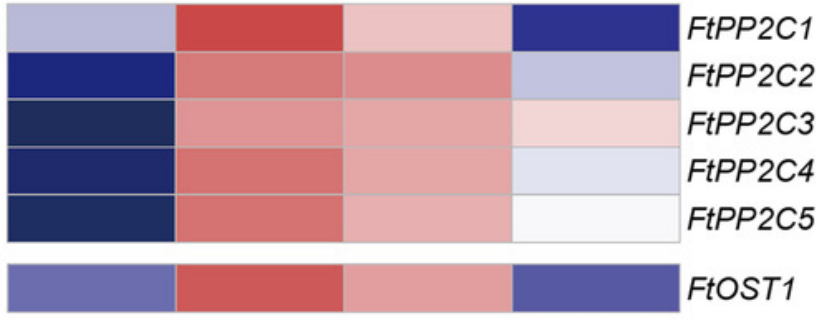

FtbZIP83

FtbZIP5 
Figure 8

Figure 8. Summary and expression patterns of TFs in response to drought stress in Tartary buckwheat.

A, Statistics of the identified TFs. B - G, Expression patterns of the large families of NAC (B), WRKY (C), AP2-EREBP (D), MYB (E), C3H (F), and bZIP (G). The values were normalized to $\log _{2}($ FPKM).
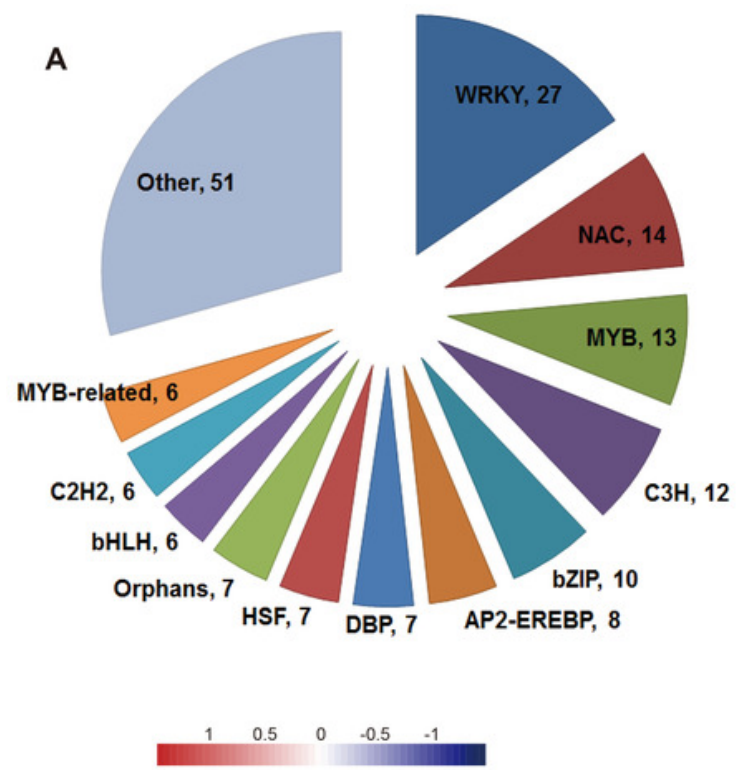

B

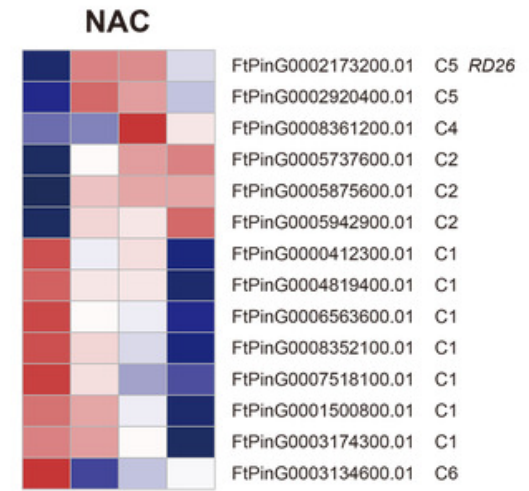

$0 \mathrm{~h} 1 \mathrm{~h} 3 \mathrm{~h} 6 \mathrm{~h}$

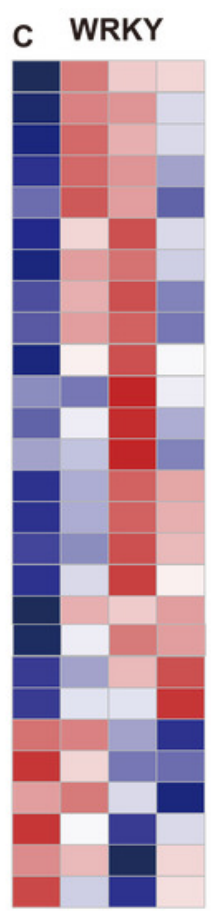

FtPinG0005751900.01 C5 FtPinG0006421500.01 C5 FtPinG0001770900.01 C5 FtPinG0003760000.01 C5 FtPinG0003981600.01 C5 FtPinG0002631400.01 C5 FtPinG0002308200.01 C5 FtPinG0008061600.01 C5 FtPinG0000782000.01 C5 FtPinG0005328500.01 C5 FtPinG0002120400.01 C4 FtPinG0006178700.01 C4 FtPinG0001713300.01 C4 FtPinG0002328400.01 C4 FtPinG0004985900.01 C4 FtPinG0005188400.01 C4 FtPinG0001576900.01 C4 FtPinG0007311700.01 C2 FtPinG0008104600.01 C2 FtPinG0008976200.01 C2 FtPinG0006100700.01 C2 FtPinG0001540600.01 C1 FtPinG0008734100.01 C1 FtPinG0002530000.01 C1 FtPinG0002848900.01 C3 FtPinG0002088200.01 C3 FtPinG0004014300.01 C3

\section{AP2-EREBP}

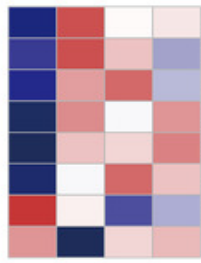

FtPinG0003023300.01 C5 FtPinG0008126800.01 C5 FtPinG0003005100.01 C5 FtPinG0008274300.01 C2 DREB1 FIPinG0003345600.01 C2 FtPinG0007618600.01 C4 DREB2 FtPinG0007073300.01 C3 FtPinG0000926400.01 C6

$0 \mathrm{~h} 1 \mathrm{~h} 3 \mathrm{~h} 6 \mathrm{~h}$
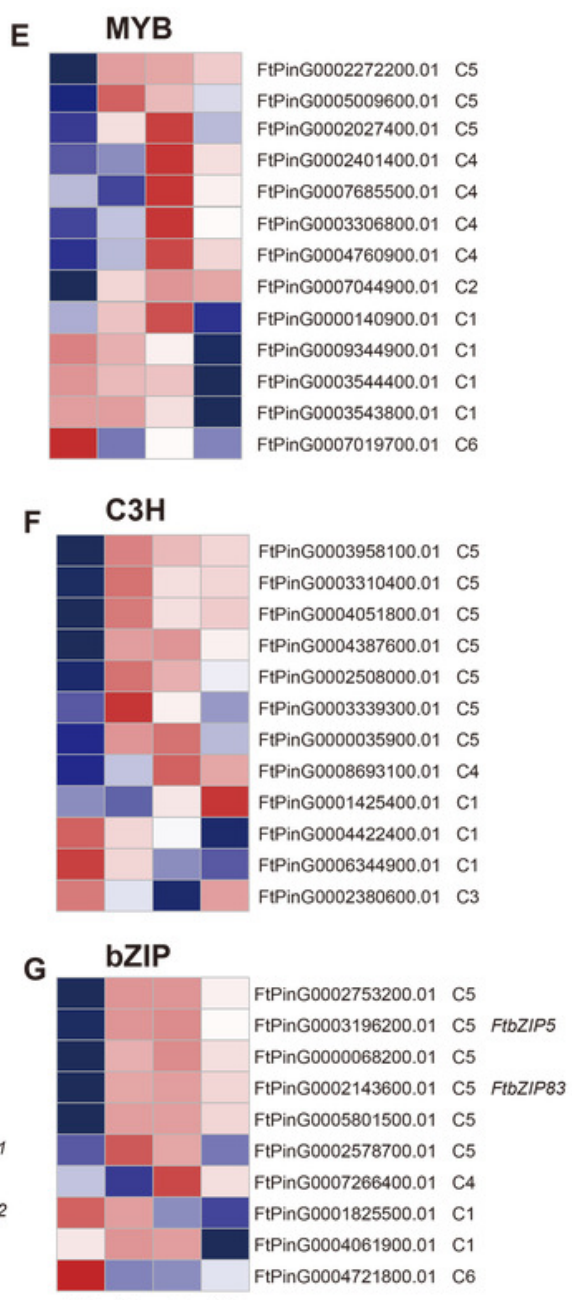

$0 \mathrm{~h} 1 \mathrm{~h} 3 \mathrm{~h} 6 \mathrm{~h}$ 
Figure 9

Figure 9. Confirmation of the transcriptome data by qRT-PCR.

A, FtPSY1. B, FtPSY2. C, FtBCH. D, FtNCED1. E, FtNCED2. F, FtNCED3. G, FtNCED4. H, FtPYR1.

I, FtPYR2. J, FtPP2C1. K, FtPP2C2. L, FtPP2C3. M, FtPP2C4. N, FtPP2C5. O, FtOST1. P, FtbZIP83.

Q, FtbZIP5. R, FtLEA1. S, FtLEA2. T, FtLEA3. U, FtLEA4. V, FtLEA5. W, FtRD29B. X, FtRD26. Y, FtDREB1D. Z, FtHB1. AA, FtHB2. BB, FtRD22. CC, FtDREB2. DD, FtRD19. EE, FtERD1. 

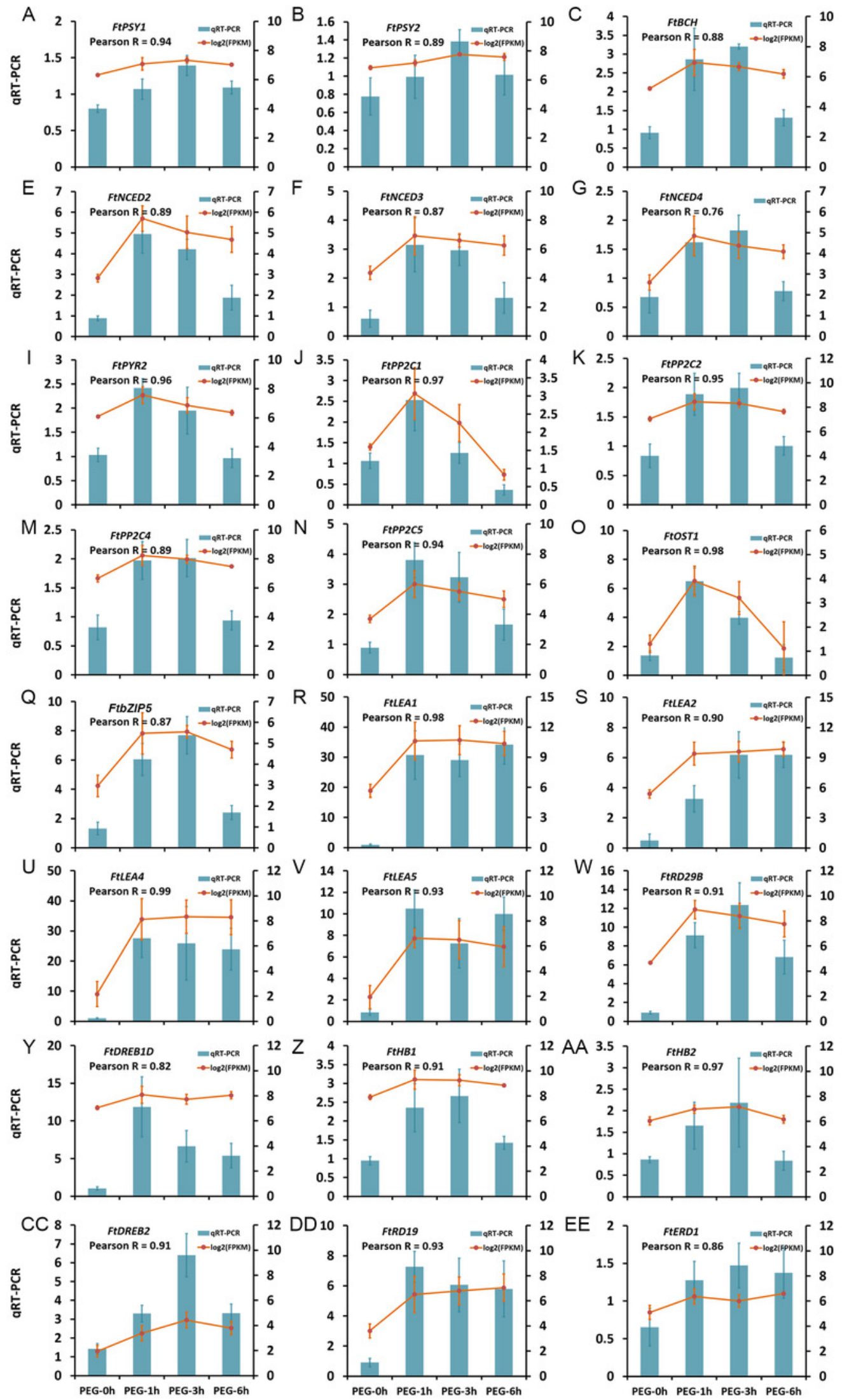
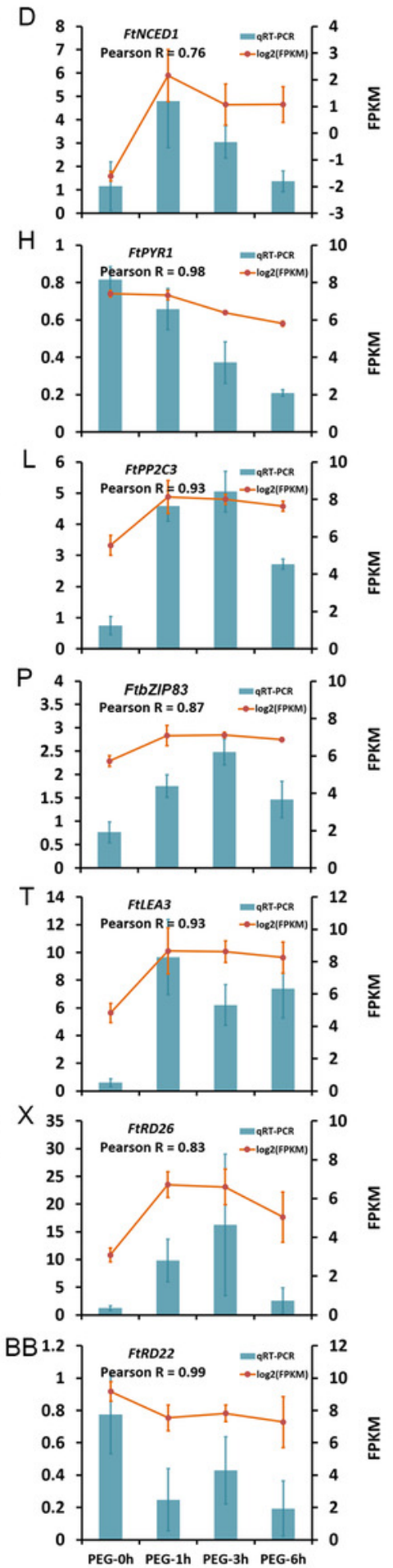


\section{Figure 10}

Figure 10. Transcriptional regulatory network in ABA-dependent and ABA-independent pathways under drought stress of Tartary buckwheat.

This figure is modified from previous studies (Fu et al. 2016; Roychoudhury et al. 2013;

Shinozaki \& Yamaguchi-Shinozaki 2007). Blue and pink rounded boxes represent genes in the ABA-dependent and ABA-independent pathway, respectively. Rounded boxes with no border indicate genes were consistent with the reported studies, whereas rounded boxes with red dotted lines indicate genes reported in previous studies were not identified in our study.

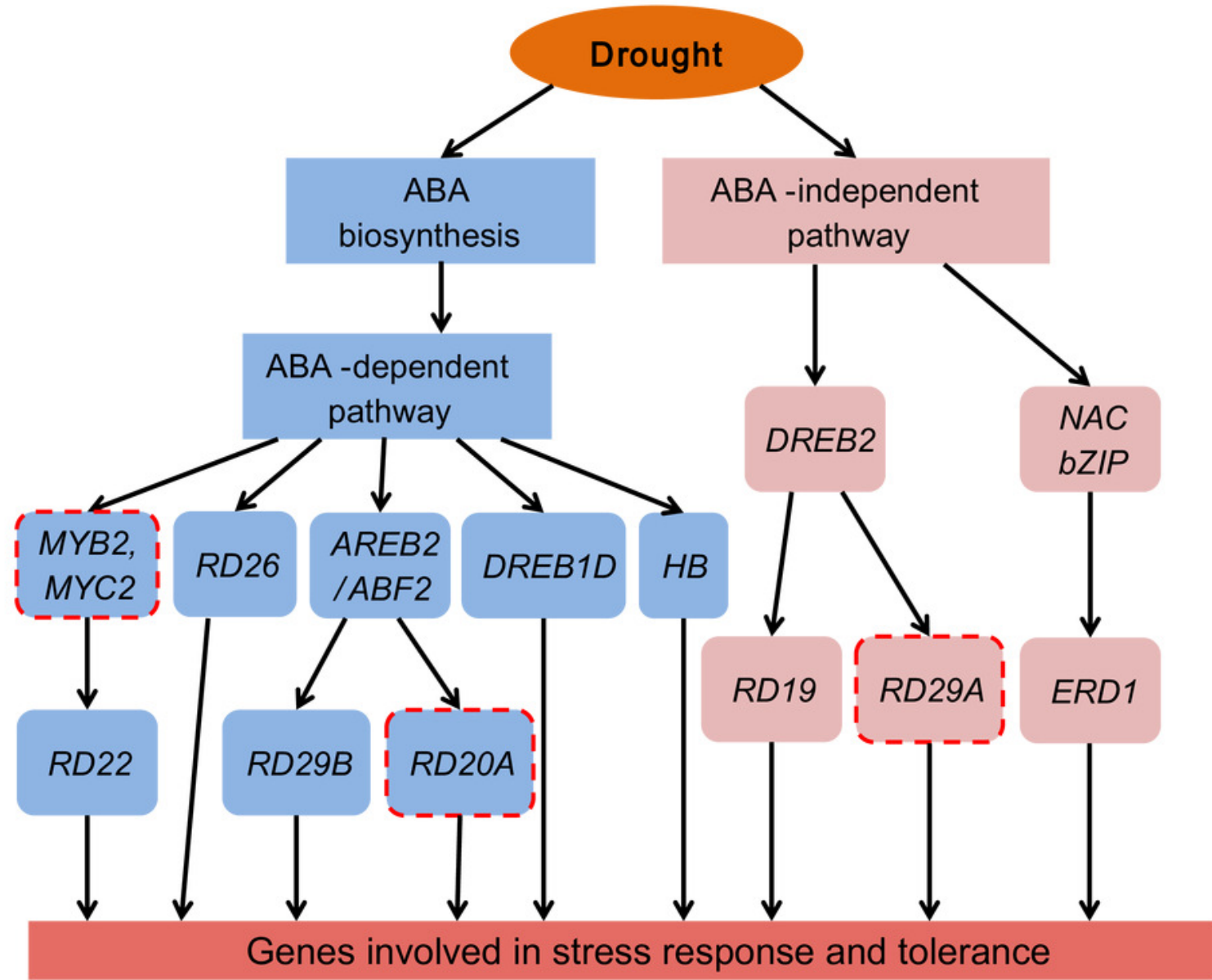




\section{Table $\mathbf{1}$ (on next page)}

Table 1. Summary of RNA-Seq results under drought tolerance of Tartary buckwheat seedlings. 
1

2 Table 1. Summary of RNA-Seq results under drought tolerance of tartary buckwheat seedlings

\begin{tabular}{|c|c|c|c|c|c|c|c|c|c|c|c|c|}
\hline \multirow{3}{*}{ Sample } & $\mathrm{PE}$ & $\mathrm{PE}$ & $P E$ & $\mathrm{PE}$ & $\mathrm{PE}$ & $\mathrm{PE}$ & $\mathrm{PE}$ & $\mathrm{PE}$ & $\mathrm{PE}$ & $\mathrm{PE}$ & $\mathrm{PE}$ & $P E$ \\
\hline & G0 & G0 & G0 & G1 & $\mathrm{G} 1$ & G1 & G3 & G3 & G3 & G6 & G6 & G6 \\
\hline & $\mathrm{h}-1$ & $\mathrm{~h}-2$ & $h-3$ & $h-1$ & $\mathrm{~h}-2$ & $h-3$ & $h-1$ & $\mathrm{~h}-2$ & $h-3$ & $\mathrm{~h}-1$ & $\mathrm{~h}-2$ & $h-3$ \\
\hline \multirow{3}{*}{$\begin{array}{c}\text { Raw } \\
\text { Reads }\end{array}$} & 45 & 45 & 46 & 47 & 62 & 48 & 48 & 44 & 47 & 49 & 50 & 44 \\
\hline & 569 & 673 & 036 & 870 & 033 & 853 & 819 & 303 & 179 & 731 & 053 & 980 \\
\hline &, 72 & ,39 &, 51 &, 57 & ,32 & ,28 & ,64 & ,64 & ,65 & ,48 & ,13 & ,06 \\
\hline Number & 4 & 0 & 8 & 0 & 8 & 2 & 4 & 0 & 6 & 6 & 8 & 8 \\
\hline \multirow{4}{*}{$\begin{array}{l}\text { Clean } \\
\text { Reads } \\
\text { Number }\end{array}$} & 42 & 42 & 43 & 45 & 59 & 46 & 45 & 42 & 44 & 47 & 47 & 42 \\
\hline & 842 & 861 & 817 & 772 & 376 & 259 & 431 & 564 & 836 & 250 & 726 & 591 \\
\hline & ,27 &, 72 & ,39 & ,02 &, 54 & ,37 & ,22 & ,42 & ,02 & ,66 & ,39 & ,28 \\
\hline & 8 & 0 & 6 & 4 & 6 & 0 & 8 & 8 & 6 & 0 & 4 & 6 \\
\hline \multicolumn{13}{|l|}{ Clean } \\
\hline Q30 & 93. & 93. & 92. & 93. & 93. & 93. & 93. & 92. & 93. & 92. & 93. & 92. \\
\hline Bases & 25 & 06 & 97 & 23 & 13 & 29 & 14 & 97 & 22 & 63 & 12 & 53 \\
\hline \multicolumn{13}{|l|}{ Rate (\%) } \\
\hline \multirow{2}{*}{$\begin{array}{c}\text { Gene } \\
\text { map Rate } \\
(\%)\end{array}$} & 74. & 74. & 75. & 74. & 74. & 74. & 74. & 75. & 74. & 75. & 74. & 75. \\
\hline & 56 & 65 & 04 & 81 & 68 & 67 & 73 & 06 & 49 & 21 & 89 & 45 \\
\hline Expresse & 24 & 24 & 24 & 24 & 24 & 24 & 24 & 24 & 24 & 24 & 24 & 24 \\
\hline d Gene & 433 & 462 & 508 & 271 & 680 & 531 & 617 & 531 & 398 & 694 & 553 & 263 \\
\hline
\end{tabular}


Table 2 (on next page)

Table 2. List of top 20 URGs and DRGs by Ih PEG treatment. 
Table 2. List of top 20 URGs and DRGs by $1 \mathrm{~h}$ PEG treatment

\begin{tabular}{|c|c|c|c|}
\hline \multicolumn{4}{|c|}{ Top 20 URGs } \\
\hline Gene_ID & Gene annotation & \multicolumn{2}{|l|}{ Log2Ra } \\
\hline FtPinG000358000 & & \multirow{2}{*}{6.97} & 2.98E- \\
\hline 0.01 & bidirectional sugar transporter N3 & & 03 \\
\hline FtPinG000365250 & & \multirow{2}{*}{6.91} & 2.23E- \\
\hline 0.01 & Embryonic protein DC-8 & & 21 \\
\hline FtPinG000120220 & & \multirow{2}{*}{6.30} & 3.66E- \\
\hline 0.01 & late embryogenesis abundant protein D-29 & & 05 \\
\hline FtPinG000208310 & & \multirow{2}{*}{5.68} & $1.81 \mathrm{E}-$ \\
\hline 0.01 & late embryogenesis abundant protein 2 & & 04 \\
\hline FtPinG000073870 & & \multirow{2}{*}{5.59} & 4.74E- \\
\hline 0.01 & uncharacterized protein & & 06 \\
\hline FtPinG000576890 & - & \multirow{2}{*}{5.36} & 3.43E- \\
\hline 0.01 & - & & 02 \\
\hline FtPinG000541900 & & \multirow{2}{*}{5.27} & 9.45E- \\
\hline 0.01 & dehydrin Rab18 & & 15 \\
\hline FtPinG000370750 & hynothetical nrotein & \multirow{2}{*}{5.21} & 2.10E- \\
\hline 0.01 & & & 03 \\
\hline FtPinG000033980 & uncharasterized nrotein & \multirow{2}{*}{4.89} & $1.48 \mathrm{E}-$ \\
\hline 0.01 & uncnaracterızea protein & & 10 \\
\hline FtPinG000070240 & late embryogenesis abundant protein D-29- & \multirow{2}{*}{4.55} & 8.81E- \\
\hline 0.01 & like & & 09 \\
\hline FtPinG000487060 & & \multirow{2}{*}{4.41} & $1.10 \mathrm{E}-$ \\
\hline 0.01 & low-temperature-induced $65 \mathrm{kDa}$ protein & & 19 \\
\hline FtPinG000272210 & 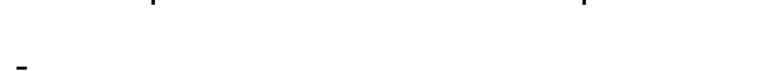 & \multirow{2}{*}{4.35} & 2.07E- \\
\hline 0.01 & - & & 03 \\
\hline FtPinG000567970 & late embryogenesis abundant protein 46- & \multirow{2}{*}{4.34} & 3.83E- \\
\hline 0.01 & like & & 11 \\
\hline FtPinG000845580 & & \multirow{2}{*}{4.31} & 3.10E- \\
\hline 0.01 & translocator protein homolog & & 07 \\
\hline FtPinG000442540 & & \multirow{2}{*}{4.29} & 1.19E- \\
\hline 0.01 & late embryogenesis abundant protein 6-like & & 03 \\
\hline FtPinG000624610 & & \multirow{2}{*}{4.29} & 7.15E- \\
\hline 0.01 & uncharacterized protein & & 06 \\
\hline FtPinG000941220 & & \multirow{2}{*}{4.15} & $1.91 \mathrm{E}-$ \\
\hline 0.01 & Carotenoid oxygenase & & 05 \\
\hline FtPinG000314640 & 11-beta-hydroxysteroid dehydrogenase 1B- & \multirow{2}{*}{3.91} & 1.85E- \\
\hline 0.01 & like & & 03 \\
\hline FtPinG000248160 & uncharacterized protein & 3.83 & 6.35E- \\
\hline
\end{tabular}


0.01

04

FtPinG000923470

$0.01 \quad$ cinnamoyl-CoA reductase 1

1.13E-

\section{Top 20 DRGs}

\begin{tabular}{|c|c|c|c|}
\hline Gene_ID & Gene annotation & $\begin{array}{l}\text { Log2Ra } \\
\text { tio }\end{array}$ & FDR \\
\hline FtPinG000782410 & & 20? & $2.50 \mathrm{E}-$ \\
\hline 0.01 & Peroxidase & $-\angle$. & 02 \\
\hline $\begin{array}{l}\text { FtPinG000979860 } \\
0.01\end{array}$ & $\begin{array}{l}\text { xyloglucan endotransglucosylase/hydrolase } \\
\text { protein } 23\end{array}$ & -2.69 & $\begin{array}{r}8.15 \mathrm{E}- \\
13\end{array}$ \\
\hline $\begin{array}{l}\text { FtPinG000328260 } \\
0.01\end{array}$ & putative peroxidase $\mathrm{N}$ & -2.57 & $\begin{array}{r}3.56 \mathrm{E}- \\
03\end{array}$ \\
\hline $\begin{array}{l}\text { FtPinG000233720 } \\
0.01\end{array}$ & Thioredoxin-like & -2.34 & $\begin{array}{r}2.00 \mathrm{E}- \\
03\end{array}$ \\
\hline $\begin{array}{l}\text { FtPinG000847030 } \\
0.01\end{array}$ & hypothetical protein & -2.19 & $\begin{array}{r}2.98 \mathrm{E}- \\
03\end{array}$ \\
\hline $\begin{array}{l}\text { FtPinG000426300 } \\
0.01\end{array}$ & probable glycosyltransferase & -1.97 & $\begin{array}{r}1.71 \mathrm{E}- \\
02\end{array}$ \\
\hline $\begin{array}{l}\text { FtPinG000551230 } \\
0.01\end{array}$ & thioredoxin-like 1-2, chloroplastic & -1.88 & $\begin{array}{r}4.90 \mathrm{E}- \\
02\end{array}$ \\
\hline $\begin{array}{l}\text { FtPinG000665130 } \\
0.01\end{array}$ & sugar transporter ERD6-like 16 & -1.86 & $\begin{array}{r}3.65 \mathrm{E}- \\
02\end{array}$ \\
\hline $\begin{array}{l}\text { FtPinG000514170 } \\
0.01\end{array}$ & ureide permease 2-like & -1.80 & $\begin{array}{r}1.86 \mathrm{E}- \\
02\end{array}$ \\
\hline $\begin{array}{l}\text { FtPinG000237630 } \\
0.01\end{array}$ & allene oxide synthase 1 , chloroplastic & -1.79 & $\begin{array}{r}1.95 \mathrm{E}- \\
02\end{array}$ \\
\hline $\begin{array}{l}\text { FtPinG000374320 } \\
0.01\end{array}$ & $\begin{array}{l}\text { indole-3-acetic acid-amido synthetase } \\
\text { GH3.6 }\end{array}$ & -1.71 & $\begin{array}{r}3.24 \mathrm{E}- \\
02\end{array}$ \\
\hline $\begin{array}{l}\text { FtPinG000679090 } \\
0.01\end{array}$ & beta-galactosidase 1 & -1.69 & $\begin{array}{r}2.98 \mathrm{E}- \\
02\end{array}$ \\
\hline $\begin{array}{l}\text { FtPinG000862160 } \\
0.01\end{array}$ & protein trichome birefringence-like 41 & -1.62 & $\begin{array}{r}1.83 \mathrm{E}- \\
03\end{array}$ \\
\hline $\begin{array}{l}\text { FtPinG000579960 } \\
0.01\end{array}$ & hypothetical protein & -1.57 & $\begin{array}{r}2.45 \mathrm{E}- \\
04\end{array}$ \\
\hline $\begin{array}{l}\text { FtPinG000496010 } \\
0.01\end{array}$ & F-box protein PP2-A12 & -1.52 & $\begin{array}{r}1.66 \mathrm{E}- \\
02\end{array}$ \\
\hline $\begin{array}{l}\text { FtPinG000959460 } \\
0.01\end{array}$ & isocitrate lyase & -1.49 & $\begin{array}{r}2.44 \mathrm{E}- \\
02\end{array}$ \\
\hline $\begin{array}{l}\text { FtPinG000647900 } \\
0.01\end{array}$ & inositol oxygenase 1-like & -1.43 & $\begin{array}{r}3.19 \mathrm{E}- \\
02\end{array}$ \\
\hline FtPinG000457590 & uncharacterized protein & -1.40 & 3.95E- \\
\hline
\end{tabular}


$\begin{array}{lr}0.01 & 02\end{array}$

FtPinG000673110

$0.01 \quad$ galactinol--sucrose galactosyltransferase 6

$-1.39 \quad 04$

FtPinG000301310

0.01 uncharacterized protein

$-1.39$

$6.45 \mathrm{E}-$

03

1

2 
Table 3 (on next page)

Table 3. List of top 20 URGs and DRGs by 3h PEG treatment. 
Table 3. List of top 20 URGs and DRGs by 3h PEG treatment

\begin{tabular}{|c|c|c|c|}
\hline \multicolumn{4}{|c|}{ Top 20 URGs } \\
\hline Gene_ID & Gene annotation & Log2Ratio & FDR \\
\hline FtPinG0001894200.01 & probable aldo-keto reductase 2 & 7.22 & 4.84E-09 \\
\hline FtPinG0003652500.01 & Embryonic protein DC-8 & 6.87 & 3.27E-07 \\
\hline FtPinG0003580000.01 & bidirectional sugar transporter N3 & 6.75 & $7.24 \mathrm{E}-15$ \\
\hline FtPinG0001202200.01 & late embryogenesis abundant protein D-29 & 6.29 & 2.90E-07 \\
\hline FtPinG0005846700.01 & uncharacterized protein & 5.80 & 7.65E-04 \\
\hline FtPinG0002083100.01 & late embryogenesis abundant protein 2 & 5.43 & 1.11E-06 \\
\hline FtPinG0000738700.01 & uncharacterized protein & 5.38 & 3.38E-09 \\
\hline FtPinG0001574200.01 & germin-like protein subfamily 1 member 13 & 5.36 & $9.63 \mathrm{E}-46$ \\
\hline FtPinG0003983300.01 & lipid transfer protein $\mathrm{H}$ & 5.35 & 4.81E-02 \\
\hline FtPinG0001106300.01 & phenylpropene reductase 2 & 5.35 & $6.21 \mathrm{E}-10$ \\
\hline FtPinG0003707500.01 & hypothetical protein & 5.16 & 7.01E-05 \\
\hline FtPinG0005419000.01 & dehydrin Rab18 & 5.07 & 4.73E-09 \\
\hline FtPinG0000702400.01 & late embryogenesis abundant protein D-29-like & 5.06 & 1.61E-03 \\
\hline FtPinG0001100200.01 & Reticulon-like protein & 4.81 & 4.21E-02 \\
\hline FtPinG0000339800.01 & uncharacterized protein & 4.76 & $1.92 \mathrm{E}-17$ \\
\hline FtPinG0005321900.01 & aquaporin TIP3-1 & 4.71 & 1.84E-02 \\
\hline FtPinG0004343900.01 & membrane protein PM19L & 4.65 & 8.39E-03 \\
\hline FtPinG0009234700.01 & cinnamoyl-CoA reductase 1 & 4.58 & 1.65E-20 \\
\hline FtPinG0006246100.01 & uncharacterized protein & 4.54 & 1.97E-05 \\
\hline FtPinG0005679700.01 & late embryogenesis abundant protein 46 -like & 4.49 & $8.46 \mathrm{E}-16$ \\
\hline \multicolumn{4}{|c|}{ Top 20 DRGs } \\
\hline Gene_ID & Gene annotation & Log2Ratio & FDR \\
\hline FtPinG0000545200.01 & omega-hydroxypalmitate O-feruloyl transferase-like & -5.67 & $5.68 \mathrm{E}-03$ \\
\hline FtPinG0007033200.01 & xyloglucan glycosyltransferase 4 & -4.94 & $9.11 \mathrm{E}-04$ \\
\hline FtPinG0008272700.01 & $A B C$ transporter $C$ family member 10 like & -3.90 & $9.18 \mathrm{E}-06$ \\
\hline FtPinG0006565000.01 & root-specific metal transporter & -3.87 & 2.81E-02 \\
\hline FtPinG0002543000.01 & unknown & -3.81 & 1.08E-02 \\
\hline FtPinG0007190100.01 & Pectinesterase inhibitor domain protein & -3.70 & 9.13E-06 \\
\hline FtPinG0006744800.01 & protein TIFY $5 \mathrm{~A}$ & -3.41 & 3.51E-02 \\
\hline FtPinG0002671400.01 & 3-ketoacyl-CoA synthase 19-like & -3.20 & 1.21E-02 \\
\hline FtPinG0007189700.01 & Pectinesterase inhibitor domain protein & -2.90 & $5.42 \mathrm{E}-03$ \\
\hline FtPinG0009444400.01 & cytochrome P450 724B1 & -2.82 & 1.01E-02 \\
\hline FtPinG0004261700.01 & putative beta-D-xylosidase & -2.82 & 1.16E-07 \\
\hline FtPinG0002765000.01 & Methyltransferase PMT5 & -2.82 & 8.12E-14 \\
\hline FtPinG0009066900.01 & alkane hydroxylase MAH1-like & -2.66 & 5.57E-05 \\
\hline FtPinG0000019300.01 & protein RADIALIS-like 5 & -2.60 & 7.23E-06 \\
\hline FtPinG0005398000.01 & cytochrome P450 76AD1-like & -2.53 & 3.62E-07 \\
\hline FtPinG0008470300.01 & hypothetical protein & -2.49 & $2.95 \mathrm{E}-09$ \\
\hline
\end{tabular}




\begin{tabular}{llrl} 
FtPinG0004039900.01 & pleiotropic drug resistance protein 3 & -2.39 & $2.00 \mathrm{E}-02$ \\
FtPinG0002454500.01 & cytosolic sulfotransferase 15-like & -2.39 & $4.25 \mathrm{E}-02$ \\
FtPinG0009798600.01 & xyloglucan endotransglucosylase/hydrolase protein 23 & -2.37 & $1.75 \mathrm{E}-11$ \\
FtPinG0001540200.01 & receptor-like protein kinase FERONIA & -2.35 & $1.75 \mathrm{E}-02$ \\
\hline
\end{tabular}

1 
Table 4 (on next page)

Table 4. List of top 20 URGs and DRGs by 6 h PEG treatment. 
Table 4. List of top 20 URGs and DRGs by 6 P PEG treatment

\begin{tabular}{|c|c|c|c|}
\hline \multicolumn{4}{|c|}{ Top 20 URGs } \\
\hline Gene_ID & Gene annotation & Log2Ratio & FDR \\
\hline FtPinG0003983300.01 & lipid transfer protein $\mathrm{H}$ & 6.95 & 3.14E-02 \\
\hline FtPinG0001202200.01 & late embryogenesis abundant protein D-29 & 6.49 & $6.21 \mathrm{E}-06$ \\
\hline FtPinG0001894200.01 & probable aldo-keto reductase 2 & 6.04 & 8.96E-06 \\
\hline FtPinG0003652500.01 & Embryonic protein DC-8 & 6.01 & 1.14E-04 \\
\hline FtPinG0005846700.01 & uncharacterized protein & 5.59 & 2.63E-03 \\
\hline FtPinG0005419000.01 & dehydrin Rab18 & 5.59 & $6.66 \mathrm{E}-13$ \\
\hline FtPinG0002083100.01 & late embryogenesis abundant protein 2 & 5.09 & $9.25 \mathrm{E}-06$ \\
\hline FtPinG0003146400.01 & 11-beta-hydroxysteroid dehydrogenase 1B-like & 4.90 & $9.80 \mathrm{E}-03$ \\
\hline FtPinG0009234700.01 & cinnamoyl-CoA reductase 1 & 4.89 & 4.45E-26 \\
\hline FtPinG0005321900.01 & aquaporin TIP3-1 & 4.71 & 1.70E-02 \\
\hline FtPinG0003580000.01 & bidirectional sugar transporter $\mathrm{N} 3$ & 4.60 & 9.39E-06 \\
\hline FtPinG0005679700.01 & late embryogenesis abundant protein 46-like & 4.60 & 5.93E-24 \\
\hline FtPinG0000702400.01 & late embryogenesis abundant protein D-29-like & 4.59 & 8.99E-03 \\
\hline FtPinG0003707500.01 & hypothetical protein & 4.50 & 1.05E-02 \\
\hline FtPinG0002481600.01 & uncharacterized protein & 4.45 & 7.07E-09 \\
\hline FtPinG0006246100.01 & uncharacterized protein & 4.45 & 1.12E-04 \\
\hline FtPinG0000835100.01 & RACK1C & 4.33 & 1.13E-04 \\
\hline FtPinG0000339800.01 & uncharacterized protein & 4.18 & $2.90 \mathrm{E}-03$ \\
\hline FtPinG0000738700.01 & uncharacterized protein & 4.11 & 3.15E-04 \\
\hline FtPinG0008455800.01 & translocator protein homolog & 3.96 & $1.18 \mathrm{E}-10$ \\
\hline \multicolumn{4}{|c|}{ Top 20 DRGs } \\
\hline Gene_ID & Gene annotation & Log2Ratio & FDR \\
\hline FtPinG0004971800.01 & methylesterase 10-like & -6.69 & 1.15E-04 \\
\hline FtPinG0006470900.01 & $(3 \mathrm{~S}, 6 \mathrm{E})$-nerolidol synthase 2 , chloroplastic/mitochondrial-like & -5.99 & 4.92E-04 \\
\hline FtPinG0008375800.01 & uncharacterized protein & -5.69 & $1.02 \mathrm{E}-02$ \\
\hline FtPinG0006565000.01 & root-specific metal transporter & -5.41 & $9.38 \mathrm{E}-03$ \\
\hline FtPinG0001164200.01 & bark storage protein A-like & -5.38 & 4.52E-02 \\
\hline FtPinG0006744800.01 & protein TIFY $5 \mathrm{~A}$ & -4.87 & 1.14E-19 \\
\hline FtPinG0007190100.01 & Pectinesterase inhibitor domain protein & -4.56 & 1.59E-07 \\
\hline FtPinG0005398000.01 & cytochrome P450 76AD1-like & -4.35 & 1.87E-33 \\
\hline FtPinG0004911500.01 & lysine-specific demethylase JMJ30-like & -4.14 & 3.11E-06 \\
\hline FtPinG0002221300.01 & hypothetical protein & -4.02 & 4.64E-20 \\
\hline FtPinG0001832800.01 & 2-isopropylmalate synthase A precursor & -3.46 & $1.25 \mathrm{E}-04$ \\
\hline FtPinG0002765000.01 & Methyltransferase PMT5 & -3.42 & $3.42 \mathrm{E}-19$ \\
\hline FtPinG0005904300.01 & lysine-specific demethylase JMJ30-like & -3.30 & 1.73E-10 \\
\hline FtPinG0006344900.01 & zinc finger $\mathrm{CCCH}$ domain-containing protein 29 & -3.26 & 1.39E-02 \\
\hline FtPinG0005602100.01 & hypothetical protein & -3.26 & 1.67E-02 \\
\hline FtPinG0000412300.01 & protein CUP-SHAPED COTYLEDON 3 & -3.21 & 7.37E-03 \\
\hline
\end{tabular}




$\begin{array}{llll}\text { FtPinG0008416800.01 } & \text { chaperone protein dnaJ C76, chloroplastic-like } & -3.19 & 6.36 \mathrm{E}-23 \\ \text { FtPinG0000385000.01 } & \text { DNA-directed RNA polymerase Il subunit RPB1 isoform X1 } & -3.15 & 6.98 \mathrm{E}-09 \\ \text { FtPinG0001825500.01 } & \text { protein FD-like } & -3.10 & 4.12 \mathrm{E}-09 \\ \text { FtPinG0009145200.01 } & \text { ABC transporter B family member 21-like } & -3.00 & 8.83 \mathrm{E}-03\end{array}$

1

2 